\title{
Effects of Repeated Stress on Age-Dependent GABAergic Regulation of the Lateral Nucleus of the Amygdala
}

\author{
Wei Zhang' and J Amiel Rosenkranz*,I \\ 'Department of Cellular and Molecular Pharmacology, The Chicago Medical School, Rosalind Franklin University of Medicine and Science, \\ North Chicago, IL, USA
}

\begin{abstract}
The adolescent age is associated with lability of mood and emotion. The onset of depression and anxiety disorders peaks during adolescence and there are differences in symptomology during adolescence. This points to differences in the adolescent neural circuitry that underlies mood and emotion, such as the amygdala. The human adolescent amygdala is more responsive to evocative stimuli, hinting to less local inhibitory regulation of the amygdala, but this has not been explored in adolescents. The amygdala, including the lateral nucleus (LAT) of the basolateral amygdala complex, is sensitive to stress. The amygdala undergoes maturational processes during adolescence, and therefore may be more vulnerable to harmful effects of stress during this time period. However, little is known about the effects of stress on the LAT during adolescence. GABAergic inhibition is a key regulator of LAT activity. Therefore, the purpose of this study was to test whether there are differences in the local GABAergic regulation of the rat adolescent LAT, and differences in its sensitivity to repeated stress. We found that LAT projection neurons are subjected to weaker GABAergic inhibition during adolescence. Repeated stress reduced in vivo endogenous and exogenous GABAergic inhibition of LAT projection neurons in adolescent rats. Furthermore, repeated stress decreased measures of presynaptic GABA function and interneuron activity in adolescent rats. In contrast, repeated stress enhanced glutamatergic drive of LAT projection neurons in adult rats. These results demonstrate age differences in GABAergic regulation of the LAT, and age differences in the mechanism for the effects of repeated stress on LAT neuron activity. These findings provide a substrate for increased mood lability in adolescents, and provide a substrate by which adolescent repeated stress can induce distinct behavioral outcomes and psychiatric symptoms.
\end{abstract}

Neuropsychopharmacology (2016) 4I, 2309-2323; doi:I0.1038/npp.2016.33; published online I6 March 2016

\section{INTRODUCTION}

Adolescence is a period of key maturational events, including ongoing refinement of numerous limbic brain regions. This maturation is sensitive to environment and experience. Experiences that occur during adolescence can thereby impart long-lasting impact. One such factor, stress during adolescence, increases the risk for psychiatric disorders (Bremner, 2003; Beardslee et al, 2012; Copeland et al, 2013). Adolescence is also unique in the symptomology of mood and anxiety disorders (Carlson and Kashani, 1988; Rao et al, 2007; Allen et al, 2010; Kessler et al, 2010), and the overall lability of mood and emotion (Dahl, 2001). These facts hint towards a uniqueness in the adolescent systems that guide mood and emotion.

The guidance and expression of moods and emotions are modulated by the activity of the basolateral complex of the amygdala (BLA). Local GABAergic circuits within the BLA

\footnotetext{
*Correspondence: Dr JA Rosenkranz, Department of Cellular and Molecular Pharmacology, The Chicago Medical School, Rosalind Franklin University of Medicine and Science, 3333 Green Bay Road, North Chicago, IL 60064, USA, Tel: + I 847578 8680, Fax: + I 847578 3268, E-mail: amiel.rosenkranz@rosalindfranklin.edu

Received 15 December 2015; revised 25 January 2016; accepted 3 February 2016; accepted article preview online 29 February 2016
}

exert ongoing inhibitory regulation when weak stimuli are present (Rainnie et al, 1991; Lang and Pare, 1997), and feedforward or feedback inhibition when activated by afferents (Lang and Pare, 1997; Isoardi et al, 2007). GABAergic inputs also synchronize projection neuron activity during behaviors that recruit the BLA, such as the acquisition and expression of learned fear (Sangha et al, 2009; Ryan et al, 2012). Loss of BLA inhibition is expected to lead to increased or disorganized BLA neuron firing along with increased or inappropriate affective responding. Consistent with this, drugs that facilitate GABAergic inhibition in the BLA are anxiolytic (Sanders and Shekhar, 1995a, b; Pidoplichko et al, 2014), while conditions with reduced inhibition within the BLA are anxiogenic (Sajdyk and Shekhar, 1997, 2000; Rainnie et al, 2004; Truitt et al, 2007, 2009; Almeida-Suhett et al, 2014) and promote generalization of BLA-dependent behaviors (Shaban et al, 2006; Lange et al, 2014). Despite known differences between adults and adolescents in amygdala-mediated affective behavior and psychiatric symptomology associated with amygdala dysfunction, little is known about differences between GABAergic regulation of the BLA between adults and adolescents. There are, however, indications that BLA GABAergic transmission does not reach mature levels until the periadolescent period (Ehrlich et al, 2013). As such, 
GABAergic inhibition within the BLA may be immature and sensitive to adolescent experience, including stress experience.

Stress typically heightens anxiety (Bremne and Vermetten, 2001; Grillon et al, 2007; Lupien et al, 2009; Pechtel and Pizzagalli, 2011), perhaps through modulation of GABAergic systems in the BLA. There are tantalizing hints that single stress (Rodriguez Manzanares et al, 2005; Isoardi et al, 2007) and repeated stress may subtly impair GABAergic regulation in the BLA of adult rodents (Reznikov et al, 2008, 2009; Gilabert-Juan et al, 2011). However, little is known about the effects of stress on GABAergic function during adolescence. There are, again, hints towards impaired GABAergic regulation of the BLA after repeated stress applied to juvenile rats that can be measured in adult rats (Jacobson-Pick and Richter-Levin, 2012; Jacobson-Pick et al, 2012; Tzanoulinou et $a l, 2014$ ) as well as changes observed as juveniles (Braga et al, 2004; Jiang et al, 2009). However, there is little direct evidence that repeated stress alters GABAergic function in the BLA, and there has been no comparison of BLA GABAergic function between adolescent exposure and adult exposure to repeated stress.

In this study we focused on the rat LAT of the BLA to examine adolescent differences in GABAergic regulation of the LAT and whether the effect of repeated restraint stress on GABAergic regulation of LAT neurons depended on the age of stress exposure.

\section{MATERIALS AND METHODS}

All experiments were approved by the Institutional Animal Care and Use Committee of Rosalind Franklin University, and conformed to the Guide for the Care and Use of Laboratory Animals (National Research Council, 2011). Measures were taken to reduce any unnecessary distress to the animals, and to reduce the total number of animals used.

\section{Subjects}

Adolescent and adult male Sprague-Dawley rats (Harlan, Indianapolis, IN) were used in this study. They were housed 2-3 per cage in the Rosalind Franklin University animal facility with free access to food and water, and maintained on a $12 \mathrm{~h}$ light/dark cycle (light cycle from 0700 to 1700 hours). After habituation to the facility, all rats underwent a 9-day stress or control handling protocol, followed by testing in the elevated plus maze (EPM) one day after the final stress/ control handling session and then electrophysiological recordings 1 day after the EPM test. Prepubescent rats arrived at the animal facility on postnatal day (PND) 25. Restraint or control handling protocols occurred PND 29-37, EPM was tested on PND 38 and electrophysiological recording were performed on PND 39. This age range was chosen to be within the prepubertal adolescence time period (Spear, 2000). Adult rats arrived on PND 58, restraint or control protocols were initiated on PND 62-65 and occurred over the next 9 days, EPM was tested PND 71-74, and electrophysiological recordings were performed on PND 72-75. This age was chosen to be postpubertal, within the adult age range. When possible ( $~ 50 \%$ of experiments), the experimenter was blind to the treatment condition. Owing to differences in size, the experimenter could not be blinded to the age condition.

\section{Repeated Restraint Protocol}

Age-matched animals were randomly assigned into either the non-restraint control or the repeated restraint group. Rats in the repeated restraint group were placed into a hemicylinder restraint tube for $20 \mathrm{~min}$ per session and 1 session per day for 7 out of 9 days in a procedure room (Rosenkranz et al, 2010; Zhang and Rosenkranz, 2012, 2013). This specific design reduces habituation to restraint, which would otherwise be significant (Zhang et al, 2014). Previous behavioral and electrophysiological studies demonstrate that this is an effective protocol to induce changes in BLA-dependent behaviors and BLA neuronal firing (Rosenkranz et al, 2010; Atchley et al, 2012; Zhang and Rosenkranz, 2012, 2013). Three different sizes of restraint hemicylinders were used to fit the size of the rats such that movement of the body was similarly restricted in adult and adolescent rats. Rats in the control group were placed into a clean polycarbonate small rodent cage with clean bedding for 20 min per session and 1 session per day for 7 out of 9 days. All the procedures were performed between 0800 to 1500 hours. Rats were run in a manner that counterbalanced age and stress groups over the course of the study.

\section{EPM Behavior Test}

To verify the effectiveness of our repeated restraint protocol, we tested animals in the EPM 1 day after the final restraint/ control handling session. The EPM was in a procedure room under dim light conditions (25-30 lux). Two sets of EPMs (Scientific Designs, Pittsburgh, PA) designed specifically for animals of different ages were used in this study (Doremus et al, 2003, 2004; Zhang and Rosenkranz, 2012); one for adult rats (open arms: 5 in width $\times 20$ in length; closed arms: 5 in width $\times 20$ in length 18 in height), and a scaled-down version for adolescent rats (open arms: 4 in width $\times 15$ in length; closed arms: 4 in width $\times 15$ in length $\times 14$ in height). Animals were placed at the junction of the four arms of the EPM, facing the open arm opposite the experimenter. Animal behavior was recorded for $5 \mathrm{~min}$ and analyzed by a personal computer (Dell E6500) running video-tracking software (Any-Maze, Stoelting, Wood Dale, IL). The number of arm entries and the proportion of time spent in the open arms (time open arms $\div$ total time $(300 \mathrm{~s})$ ) were calculated.

\section{Adrenal Gland Weight}

A secondary measure of the effectiveness of repeated stress is by measurement of relative adrenal gland weight. Adrenal glands were excised bilaterally from a subset of rats after being killed. Adrenal weight was measured while wet. Adrenal weight was normalized to body weight of the rat (adrenal weight $\div$ body weight) and compared across groups.

\section{In Vivo Extracellular Recording}

One day after the EPM behavioral test, in vivo extracellular recordings were performed in anesthetized rats (urethane, $1.5 \mathrm{~g} / \mathrm{kg}$ dissolved in $0.9 \%$ saline, intraperitoneally). Rats 
were placed in a stereotaxic device (Stoelting, Wood Dale, IL) after deep anesthesia was confirmed. Their body temperature was monitored via a rectal temperature probe, and maintained at $36-37^{\circ} \mathrm{C}$ using a heating pad with a temperature controller (Model TC-1000; CWE, Ardmore, PA). The amygdala was localized using a stereotaxic atlas (Paxinos and Watson, 2008). For adult rats, the coordinates used for the amygdala centered on $4.8-5.2 \mathrm{~mm}$ lateral from midline, and $2.5-3.8 \mathrm{~mm}$ caudal from bregma. For adolescent rats, coordinates were scaled according to the measured distance between the bregma and interaural skull landmarks. Burr holes were drilled in the skull bilaterally at locations overlying the LAT. The hole overlying one amygdala was used for fixing a screw for EEG recording. In some experiments, a cannula for infusions (pulled glass pipette with shank diameter $50-100 \mu \mathrm{m}$ ) was lowered into the middle of the LAT ( $15^{\circ}$ angle off the rostral-caudal axis). Single-barrel recording electrodes were constructed from glass pipettes (World Precision Instruments, Sarasota, FL), pulled using a vertical microelectrode puller (PE-2; Narishige, Tokyo, Japan), and broken under a microscope to produce a $1-2 \mu \mathrm{m}$ diameter tip. The recording electrode was filled with 2\% Pontamine Sky Blue (Sigma-Aldrich, St Louis, $\mathrm{MO}$ ) in $2 \mathrm{M} \mathrm{NaCl}$ and then slowly lowered into the amygdala via a hydraulic microdrive (Model MO-10; Narishige). During extracellular recording, signals were amplified with a headstage connected to a preamplifier (2400 Amplifier; Dagan Corporation, Minneapolis, MN), filtered at $0.3 \mathrm{~Hz}$ (low cutoff frequency) and $3 \mathrm{kHz}$ (high cutoff frequency), and outputted simultaneously to an oscilloscope (Model 2532; BK Precision, Yorba Linda, CA) and an audio monitor (Model AM8; Grass Instruments, West Warwick, RI). In addition, amplified outputs were digitized $(10 \mathrm{kHz}$; Model ITC-18; HEKA, Bellmore, NY) and fed to a personal computer (Mac Pro/2.8; Apple, Cupertino, CA), monitored using the Axograph X software and stored on a hard disk for off-line analysis. Throughout the experiment, the anesthesia state of the animal was monitored via cortical EEG. Animals were considered under deep anesthesia when the EEG displayed a slow $(0.5-1.2 \mathrm{~Hz})$ rhythmic waveform.

Local application of drugs. In some experiments, intraLAT infusions were performed during electrophysiological recordings. Infusions of either artificial cerebrospinal fluid (ACSF, prepared as below) or picrotoxin (in ACSF, $10 \mathrm{pmol} /$ $100 \mathrm{nl} / 5 \mathrm{~min}$; Ascent Scientific, Princeton, NJ) were delivered through the cannula (Picopump 1400 pressure ejector; World Precision Instruments) after isolation of a single LAT neuron to measure the effects on firing rate in a withinneuron design. To provide additional data, spontaneously firing neurons in a single track were recorded before infusions and in another track after infusions (counterbalanced between experiments) to measure the effects on the number of neurons per electrode penetration track.

Ionotophoretic application of GABA and glutamate. To examine the response of LAT neurons to GABA or glutamate, in vivo microiontophoresis was coupled to electrophysiological recording. Multibarrel microelectrodes (4 barrels; A-M Systems, Sequim, WA) were constructed using a vertical microelectrode puller (PE-2; Narishige), and the tip was broken back under microscopic guidance. One barrel of the microelectrode was filled with $2 \%$ Pontamine Sky Blue (Sigma-Aldrich) in $2 \mathrm{M} \mathrm{NaCl}$ (Fisher Scientific, Pittsburgh, PA) for electrophysiological recordings and the second barrel was filled with $1 \mathrm{M} \mathrm{NaCl}$ for automatic current balancing. The remaining barrels were used for drug application, and were filled with $50 \mathrm{mM}$ glutamate $(\mathrm{pH}$ 8.0; Alfa Aesar, Ward Hill, MA) or $100 \mathrm{mM}$ GABA (pH 4.0; Ascent Scientific). All of the drugs were dissolved in $20 \mathrm{mM}$ $\mathrm{NaCl}$ solution. Glutamate was ejected with anodal iontophoretic current, and GABA was ejected with cathodal iontophoretic current (E104B; Fintronics). Retaining currents of the opposite polarity were used $(10 \mathrm{nA})$. In some experiments, the glutamate ejection current was adjusted to maintain a firing rate of BLA projection neurons near $5 \mathrm{~Hz}$. After a stable firing rate was achieved with glutamate iontophoresis, GABA was coiontophoresed at ascending current amplitudes (5-40 nA, $20 \mathrm{~s}$ duration each). In separate experiments, glutamate alone was iontophoresed at ascending current amplitudes (10-40 nA). The number of action potentials in response to glutamate ejection and during GABA coiontophoresis was measured.

Histology for in vivo experiments. At the conclusion of experiments, Pontamine was ejected from the recording electrode $(-30 \mu \mathrm{A}, 20 \mathrm{~min})$ and high-frequency current was passed through the cannula, if applicable, to mark its position. Rats were decapitated and their brains were removed and stored in $4 \%$ paraformaldehyde (SigmaAldrich) in $0.1 \mathrm{M}$ phosphate-buffered saline (PBS) overnight, and then cryoprotected in 25\% sucrose (Sigma-Aldrich) in $0.1 \mathrm{M}$ PBS. Brains were sliced into $60-\mu \mathrm{m}$-thick sections using a freezing microtome (Leica Microsystems, Buffalo Grove, IL) and stained with cresyl violet. Recording sites were verified under light microscopy. The histological section with greatest Pontamine deposit was matched to atlas representations of the LAT. The recording sites were reconstructed in atlas representations based on this Pontamine deposit. Recording sites that were found to lie outside the LAT were not included in data analysis.

Analysis of extracellular recordings. Putative projection neurons and interneurons were separated based on previously established criteria that us action potential width (Rosenkranz and Grace, 1999; Likhtik et al, 2006; Zhang and Rosenkranz, 2012). However, because these characteristics can vary depending on electrode and filter settings, these criteria were retested here by measuring the half-width of action potentials and plotting a frequency distribution of half-widths. The best-fit for this distribution was tested between one second-order polynomial (indicative of a single population) and two second-order polynomials (indicative of two populations). The half-width at which two polynomials intersect was used as the cutoff point to separate between a population with narrow action potentials and a population with wider action potentials. The intersection was $0.225 \mathrm{~ms}$. To reduce uncertainty, a $0.015 \mathrm{~ms}$ buffer on either side of this intersection (0.205-0.240 ms) was added, and neurons within this window were excluded.

Projection neurons were included in analysis if they met the following criteria: they were located within the LAT as 
determined by reconstruction based on histological staining, action potentials had a signal-to-noise ratio $>3: 1$, the firing rate was stable, and the action half-width was $>0.240 \mathrm{~ms}$ (Rosenkranz and Grace, 1999; Zhang and Rosenkranz, 2012). The spontaneous firing rate was measured as the number of action potentials/s $(\mathrm{Hz})$ over a minimum of $4 \mathrm{~min}$. The neurons/track was measured as the number of neurons encountered (ie stable firing rate data obtained) during an electrode penetration through the LAT. In all instances, this was a penetration through the entire depth of the LAT. However, because the LAT size is different across age, these data were normalized by the length of the penetration (ie length of the LAT at that penetration site) to produce a measure of active neurons per $\mathrm{mm}$.

Iontophoresis. The effect of GABA iontophoresis was calculated using the following equation: ((number of $\mathrm{AP}_{\text {Glutamate }+\mathrm{GABA}}$ - number of $\left.\mathrm{AP}_{\text {Glutamate }}\right) /$ number of $\mathrm{AP}_{\mathrm{Glu}}$ tamate) $\times 100$, where APGlutamate is the number of action potentials during glutamate iontophoresis, and $\mathrm{AP}_{\mathrm{Glutamate}+\mathrm{GABA}}$ is the number of action potentials during glutamate and GABA coiontophoresis.

\section{In Vitro Whole-Cell Recordings}

In vitro whole-cell recordings were obtained from brain slices 2 days after the final restraint or control session. Chemicals were from Fisher Scientific (Pittsburgh, PA), with the exception of sucrose, ascorbic acid and sodium pyruvate, potassium gluconate, EGTA, HEPES, Mg-ATP, Tris-GTP, and phosphocreatine, which were from Sigma-Aldrich. All other exceptions are indicated.

Slice preparation. Rats were anesthetized by intraperitoneal injection of ketamine (90 mg/kg; Vedco, St Joseph, MO) and xylazine $(10 \mathrm{mg} / \mathrm{kg}$; Lloyd Laboratories, Shenandoah, IA). Rats were perfused transcardially with an ice-cold solution containing (in mM) $2.5 \mathrm{KCl}, 1.25 \mathrm{NaH}_{2} \mathrm{PO}_{4}, 25$ $\mathrm{NaHCO}_{3}, 7$ dextrose, $7 \mathrm{MgCl}_{2}, 0.5 \mathrm{CaCl}_{2}, 210$ sucrose, 1.3 ascorbic acid, and 3 sodium pyruvate, saturated with a $95 / 5 \%$ $\mathrm{O}_{2} / \mathrm{CO}_{2}$ gas mixture, with an osmolality of $290-300 \mathrm{mOsm}$. After perfusion, the rat was quickly decapitated and the brain was removed and sliced in $300 \mu \mathrm{m}$ sections in the same solution (Vibratome Series 1000; Vibratome, St Louis, MO). Brain slices were then incubated at $34^{\circ} \mathrm{C}$ for $45 \mathrm{~m}$ in a solution containing (in $\mathrm{mM}$ ) $125 \mathrm{NaCl}, 2.5 \mathrm{KCl}, 1.25$ $\mathrm{NaH}_{2} \mathrm{PO}_{4}, 25 \mathrm{NaHCO}_{3}, 10$ dextrose, $1 \mathrm{MgCl}_{2}, 2 \mathrm{CaCl}_{2}, 1.3$ ascorbic acid, and 3 pyruvic acid, saturated with a 95/5\% $\mathrm{O}_{2} / \mathrm{CO}_{2}$ gas mixture. After $45 \mathrm{~m}$, slices sat at room temperature until whole-cell recordings.

Whole-cell recording. Brain slices were transferred to a recording chamber perfused with a solution containing (in mM) $125 \mathrm{NaCl}, 2.5 \mathrm{KCl}, 1.25 \mathrm{NaH}_{2} \mathrm{PO}_{4}, 25 \mathrm{NaHCO}_{3}, 10$ dextrose, $1 \mathrm{MgCl}_{2}$ and $2 \mathrm{CaCl}_{2}$, saturated with a $95 / 5 \%$ $\mathrm{O}_{2} / \mathrm{CO}_{2}$ gas mixture. Patch electrodes (borosilicate glass, outer diameter $1.5 \mathrm{~mm}$ and inner diameter $0.86 \mathrm{~mm}$; Sutter Instruments, Novato, CA) were pulled (1.5-4 $\mathrm{M} \Omega$ open-tip resistance; Flaming/Brown micropipette puller, Model P-97; Sutter Instruments) and filled with a solution containing (in $\mathrm{mM}$ ) $120 \mathrm{~K}$-gluconate, $20 \mathrm{KCl}, 0.2 \mathrm{EGTA}, 10 \mathrm{HEPES}$, and
$2 \mathrm{NaCl}$, with a $\mathrm{pH}$ of $7.3,280-290 \mathrm{mOsm}$. On the day of recording, $4 \mathrm{mM}$ Mg-ATP, $0.3 \mathrm{mM}$ Tris-GTP, $7 \mathrm{mM}$ phosphocreatine, and $0.2 \%$ neurobiotin (Vector Laboratories, Burlingame, CA) were added to the internal recording solution. During whole-cell recording, neurons were visualized under IR-DIC illumination using a fixed-stage upright microscope (BX51WI; Olympus). Whole-cell recordings were performed at $31-34^{\circ} \mathrm{C}$. GABA receptor-mediated spontaneous inhibitory postsynaptic currents (sIPSCs) were isolated using D-2-amino-5-phosphonovalerate (D-APV, $50 \mu \mathrm{M}$ dissolved in $100 \mathrm{mM} \mathrm{NaOH}$; Abcam Biochemicals, Cambridge, MA) and 6-cyano-7- nitroquinoxaline-2, 3-dione (CNQX, $10 \mu \mathrm{M}$; dissolved in double-distilled water; Ascent Scientific), to block NMDA receptors and $\alpha$-amino-3hydroxy-5-methyl-4-isoxazole propionic acid (AMPA) receptors. Miniature inhibitory postsynaptic currents (mIPSCs) were isolated using APV, CNQX, and the addition of the sodium channel blocker tetrodotoxin citrate (TTX, $1 \mu \mathrm{M}$; Ascent Scientific). Miniature excitatory postsynaptic currents (mEPSCs) were isolated using TTX $(1 \mu \mathrm{M})$, and (+)-bicuculline $(10 \mu \mathrm{M}$; Ascent Scientific; dissolved in dimethyl sulfoxide) and picrotoxin $(10 \mu \mathrm{M}$; dissolved in ethanol) to block $\mathrm{GABA}_{\mathrm{A}}$ receptors. Final solvent concentrations were $<0.1 \%$ of the total ACSF volume. The frequency and amplitude of sIPSCs, mIPSCs, and mEPSCs were recorded with the membrane potential held at -70 to $-80 \mathrm{mV}$.

In some experiments, a bipolar stimulation electrode was placed $50-100 \mu \mathrm{m}$ from the soma of the recorded neuron. EPSCs were evoked by stimulation $(0.1 \mathrm{~ms}$ duration) and measured in the presence of picrotoxin $(10 \mu \mathrm{M})$, bicuculline $(10 \mu \mathrm{M}), \mathrm{D}-\mathrm{APV}(50 \mu \mathrm{M})$ and CGP55845 $(0.5 \mu \mathrm{M})$ to isolate the AMPA receptor-mediated component. A current intensity was used to evoke an EPSC between 50and $125 \mathrm{pA}$. The paired-pulse response was measured with an interstimulus interval of $50 \mathrm{~ms}$.

Signals were amplified by a headstage (HS-2A; Molecular Devices, Sunnyvale, CA) and fed to an AxoClamp 2A amplifier (Molecular Devices). Amplified outputs were filtered at $1 \mathrm{kHz}$ and digitized (10 kHz, ITC-18; HEKA), and then transmitted to and stored on an Apple Computer (MacPro; Apple) running the AxoGraph $\mathrm{X}$ software (Axograph Scientific). Neurons were classified as LAT projection neurons and used for analysis if they (1) were histologically confirmed to lie within the LAT, (2) had a morphology consistent with projection neurons (McDonald, 1982; McDonald, 1992), and (3) had a resting membrane potential at least $-60 \mathrm{mV}$.

Histology for in vitro experiments. After recordings, slices were fixed in $4 \%$ paraformaldehyde in $0.1 \mathrm{M}$ PBS for up to 4 weeks at $4{ }^{\circ} \mathrm{C}$. Sections were rinsed three times with PBS, treated with Triton X-100 (VWR International, Radnor, PA; $1 \%$ in PBS) for 6 to $8 \mathrm{~h}$, and then incubated in Vectastain ABC Reagent (Vector Laboratories) in PBS at room temperature overnight. After three rinses with PBS, sections were reacted with diaminobenzidine and $\mathrm{H}_{2} \mathrm{O}_{2}$ (Peroxidase Substrate Kit, Vector Laboratories) to visualize the neurobiotin-filled neurons. Sections were washed in PBS repeatedly to stop the reaction. Sections were mounted, dried, and coverslipped. Stained sections were used to localize the recording sites, verified by the position of the neurobiotin-filled neurons. 
Analysis of whole-cell recordings. Resting membrane potential in voltage-clamp mode was determined immediately after break-in as the voltage when zero current was recorded. Synaptic events were detected using a variable amplitude sliding template with the shape of an average synaptic current (Axograph X). The detection threshold for the events was set at 2.5 to 3 times baseline noise standard deviation. The frequency and amplitude of synaptic events was measured. Paired-pulse ratio of evoked EPSCs was measured as $\mathrm{P} 2 / \mathrm{P} 1$, where $\mathrm{P} 2$ is the EPSC response to pulse 2 and $\mathrm{P} 1$ is the EPSC response to pulse 1.

\section{Statistical Analysis}

Statistical tests were performed using the Prism 5 software (GraphPad, La Jolla, CA). A $p$-value $<0.05$ was considered statistically significant. When two groups were subjected to a planned comparison, data were tested for normal distribution (D'Agostino and Pearson normality test) and compared with a two-tailed unpaired $t$-test. When multiple factors were analyzed, measures were compared using a two-way
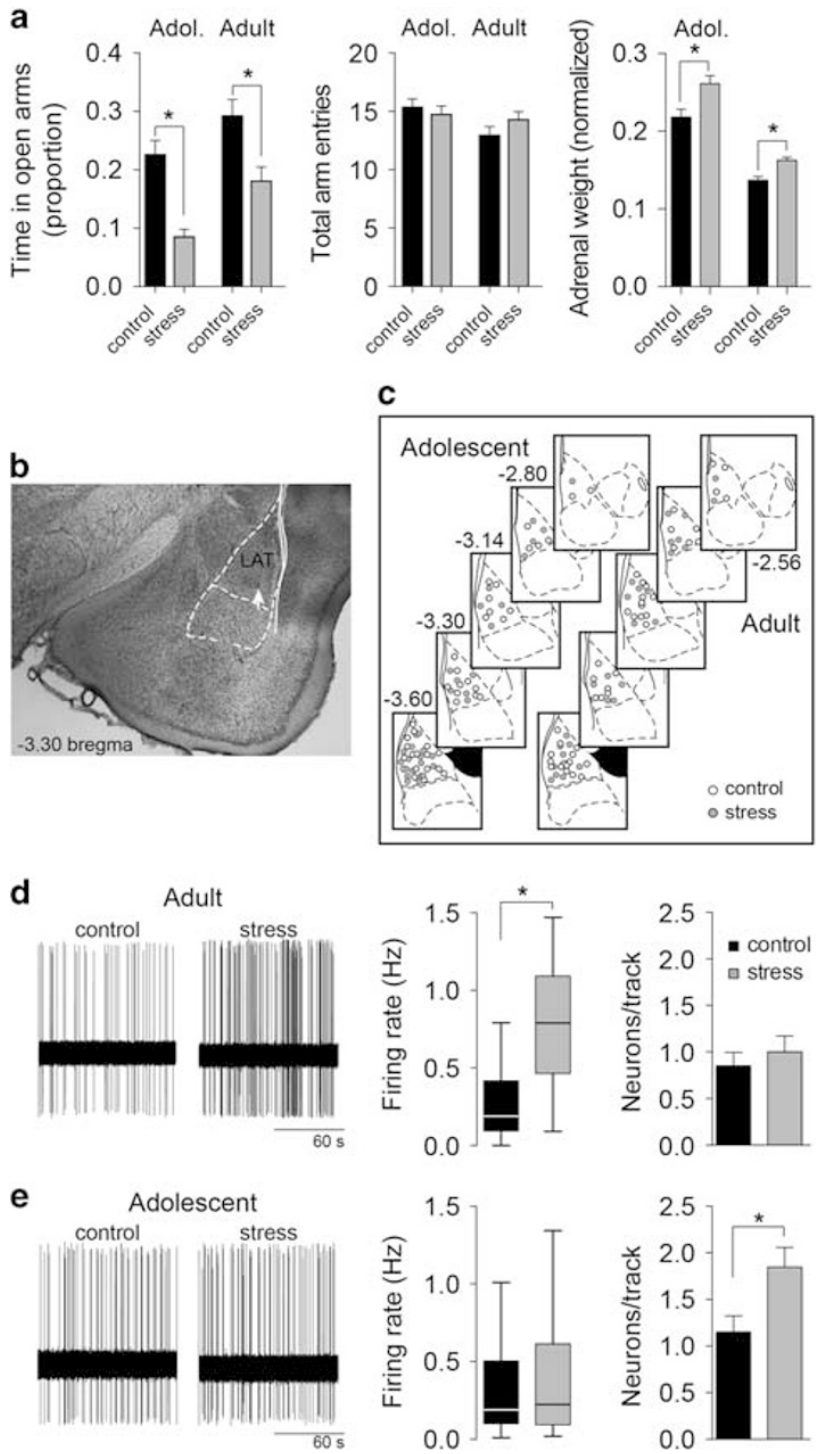

ANOVA with group (control or repeated restraint) and age (adolescent or adult) as primary factors. Where indicated, treatment (eg picrotoxin) or current were used as repeatedmeasures factors in two-way repeated-measures ANOVA. Holm-Sidak's multiple comparisons test was used for further comparison when a significant difference was found in ANOVA. In some experiments, where noted, the magnitude of the impact of stress was measured. This was accomplished by normalization of stress values by the average control values. All data were presented as mean \pm SEM, unless otherwise specified.

\section{RESULTS}

\section{Effectiveness of Repeated Restraint Stress}

The time spent on the open arms of the EPM was used as an index of anxiety-like behavior to confirm the effectiveness of the repeated stress procedure. Similar to previous studies, repeated restraint stress decreased exploration of the open arms (Figure 1a; main effect of stress $\mathrm{F}(1,198)=26.1$, $p<0.0001$, two-way ANOVA), with significant decreases in adult rats (percent of time in open arm, control $29.2 \pm 2.8 \%$, $n=56$ rats; stress $18.0 \pm 2.5 \%, n=59$ rats; $p<0.05$, Holm-Sidak's multiple comparisons test) and adolescent rats (control $22.5 \pm 2.5 \%, n=41$; stress $8.6 \pm 1.4 \%, n=46$; $p<0.05$, Holm-Sidak's multiple comparisons test). There was no significant age $\times$ stress interaction $(\mathrm{F}(1,198)=0.345$, $p=0.557$, two-way ANOVA), indicating that stress had similar effects in adult and adolescent open-arm exploration under these conditions. There was no significant effect of stress on the total number of arm entries (main effect of stress $\mathrm{F}(1,198)=0.23, p=0.63)$. This provides behavioral

\footnotetext{
Figure I Dissociable effect of repeated stress on the firing of lateral nucleus (LAT) neurons in adults and adolescents. (a) To verify the effectiveness of repeated restraint stress, behavior in the elevated plus maze was measured. Repeated stress significantly decreased the proportion of time in the open arms of the elevated plus maze (EPM) (left) in adolescent and adult rats (* $p<0.05$, Holm-Sidak's multiple comparisons test after twoway analysis of variance (ANOVA)). There was no significant effect of repeated stress on total arm entries ( $p>0.05$, two-way ANOVA). Repeated stress also increased the weight of adrenal glands (normalized to body weight) in adult and adolescent rats $(p<0.05$, Holm-Sidak's multiple comparisons test after two-way ANOVA), a secondary measure of the effectiveness of the stressor protocol. (b) Only neurons that were histologically verified to reside within the LAT were included for analysis. Neurons were localized by reconstruction based on location of Pontamine Sky Blue. Shown here is an example of a Pontamine Sky Blue deposit (white arrow) in the LAT. (c) The location of neurons from adolescent (left) and adult rats (right) included for analysis spanned the LAT in control (white circles) and repeated stress conditions (gray circles). (d) The firing rate of LAT neurons was measured from rats exposed to repeated stress or control handling (left). In adult rats, repeated stress significantly increased the firing rate of LAT neurons (box plot \pm Tukey, ${ }^{*} p<0.05$, Holm-Sidak's multiple comparisons test after two-way ANOVA), with no effect on the number of firing neurons encountered per electrode penetration track (right, $p>0.05$, Holm-Sidak's multiple comparisons test after two-way ANOVA). (e) In adolescent rats, repeated stress did not significantly increase the firing rate of LAT neurons (box plot \pm Tukey, $p>0.05$, Holm-Sidak's multiple comparisons test after two-way ANOVA), but significantly increased the number of firing neurons encountered per electrode track (right, $* p<0.05$, HolmSidak's multiple comparisons test after significance in two-way ANOVA).
} 

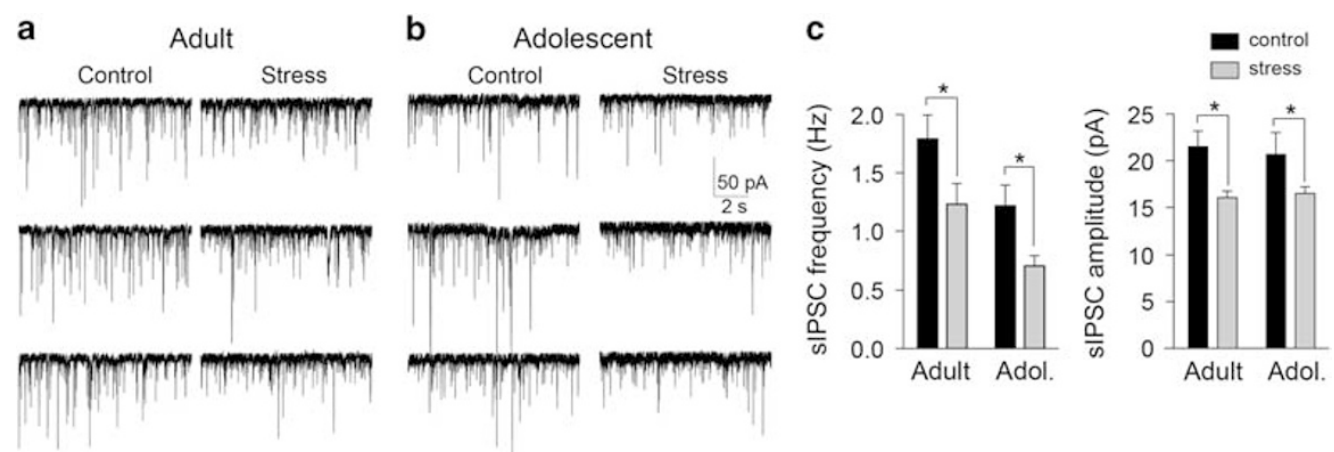

Figure 2 Repeated stress decreases GABAergic inhibition. Spontaneous inhibitory postsynaptic current (sIPSCs) were measured from lateral nucleus (LAT) principal neurons ex vivo to gauge ongoing GABAergic inhibition from synaptic sources. (a) Repeated stress decreased the frequency of sIPSCs in this example from an adult rat. (b) Repeated stress decreased the frequency of sIPSCs in this example from an adolescent rat. (c) Repeated stress significantly decreased the frequency of sIPSCs in adult and adolescent rats (left; * $p<0.05$. Holm-Sidak's multiple comparisons test after significance in two-way analysis of variance (ANOVA). Repeated stress significantly decreased the amplitude of sIPSCs in adult and adolescent rats (right; * $p<0.05$, Holm-Sidak's multiple comparisons test after significance in two-way ANOVA).

evidence for the effectiveness of the repeated restraint protocol. A second confirmation for the effectiveness of repeated stress was obtained by measurement of adrenal gland weight. Repeated stress increased the relative adrenal weight (normalized to body weight; main effect of stress, $\mathrm{F}(1,52)=18.72, p<0.0001$, two-way ANOVA, $n=14$ rats per group) in both adult and adolescent rats (control compared with stress, $p<0.05$, Holm-Sidak's multiple comparisons test for both adult and adolescents).

\section{Different Effects of Stress on LAT Neuron Firing in Adolescent Rats}

Firing rate and the number of active neurons are measures of LAT neuronal activity. Neuronal activity was measured from neurons histologically verified to reside within the LAT (Figures $1 \mathrm{~b}$ and $\mathrm{c}$ ). Repeated stress increased the overall firing rate of LAT projection neurons in adult rats but not in adolescent rats (Figures $1 \mathrm{~d}$ and e; main effect of stress, $\mathrm{F}(1,141)=28.32, p<0.0001$; stress $\times$ age interaction, $\mathrm{F}(1,141)=12.12, p=0.0007 ; p<0.05$, Holm-Sidak's multiple comparisons test in adults but not in adolescents; adolescent control $n=41$ neurons, adolescent stress $n=32$ neurons, adult control $n=40$ neurons, adult stress $n=32$ neurons). Repeated stress increased the number of active neurons encountered in adolescent rats, but not in adult rats (Figures 1d and e; main effect of stress, $\mathrm{F}(1,153)=5.676$, $p=0.0184 ; p<0.05$, Holm-Sidak's multiple comparisons test in adults but not in adolescents; adolescent control $n=40$ tracks, adolescent stress $n=39$ tracks, adult control $n=40$ tracks, adult stress $n=39$ tracks). The size of the LAT is slightly smaller in adolescent rats (Chareyron et al, 2012). Therefore, we normalized neurons/track by the track distance, and compared neurons per $\mathrm{mm}$. This analysis produced similar results, with a significant effect of stress that depended on age (stress $\times$ age interaction, $\mathrm{F}(1,153)=4.127, p=0.044)$. Repeated stress increased the number of neurons per $\mathrm{mm}$ in adolescent rats (control $1.7 \pm 0.2$ neurons per $\mathrm{mm}$, stress $2.9 \pm 0.3$ neurons per $\mathrm{mm}$; $p<0.05$, Holm-Sidak's multiple comparisons test) but not in adult rats (control $1.1 \pm 0.2$ neurons per mm, stress $1.3 \pm 0.2$ neurons per mm; $p>0.05$, Holm-Sidak's multiple comparisons test). While trending, there was no significant difference between adult and adolescent controls in the number of neurons per mm ( $p>0.05$, Holm-Sidak's multiple comparisons test). These data indicate that repeated stress has different effects on the neuronal activity of LAT neurons in adult and adolescent rats (Zhang and Rosenkranz, 2012). LAT neuronal activity is highly sensitive to GABAergic regulation. These differences in firing may reflect distinct effects of stress on GABAergic regulation in adult and adolescents, which will be tested below.

\section{Different Effects of Stress on GABAergic Regulation of Adolescent LAT Neurons In Vitro}

To test whether repeated stress alters GABAergic function in the LAT, in vitro measures of GABAergic synaptic transmission were obtained. sIPSCs were measured to assess spontaneous GABA transmission. Repeated stress decreased the frequency of sIPSCs (main effect of stress $\mathrm{F}(1,173)=10.4$, $p=0.0015$, two-way ANOVA) in both adult (Figures 2a and c; control $1.8 \pm 0.20 \mathrm{~Hz}, n=44$ neurons; stress $1.2 \pm 0.18 \mathrm{~Hz}$, $n=38$ neurons; $p<0.05$, Holm-Sidak's multiple comparisons test) and in adolescent rats (Figures $2 \mathrm{~b}$ and c; control $1.2 \pm 0.18 \mathrm{~Hz}, \quad n=42$ neurons; stress $0.7 \pm 0.09, \quad n=53$ neurons; $p<0.05$, Holm-Sidak's multiple comparisons test). There was a significant effect of stress on the amplitude of sIPSCs (main effect of stress $\mathrm{F}(1,173)=9.0, p=0.003$, two-way ANOVA), with a significant decrease of sIPSC amplitude in adult (Figure 2c; control $21.5 \pm 1.7 \mathrm{pA}$, stress $16.1 \pm 0.7 \mathrm{pA} ; p<0.05$, Holm-Sidak's multiple comparisons test) and in adolescent rats (control $20.7 \pm 2.4 \mathrm{pA}$, stress $16.5 \pm 0.7 \mathrm{pA} ; \quad p<0.05, \quad$ Holm-Sidak's multiple comparisons test).

The frequency of sIPSCs reflect a combination of factors that regulate $\mathrm{GABA}$ release and action potential firing of GABAergic neurons. To measure factors specific to GABA release, mIPSCs were measured. The effect of stress on mIPSCs depended on age (stress $\times$ age interaction $\mathrm{F}(1,105)=4.2, p=0.04$, two-way ANOVA). Stress significantly decreased the frequency of mIPSCs in adolescent (Figures $3 \mathrm{~b}$ and c; control $0.88 \pm 0.12 \mathrm{~Hz}, n=27$ neurons; stress $0.49 \pm 0.11 \mathrm{~Hz}, n=31$ neurons; $p<0.05$, Holm-Sidak's 


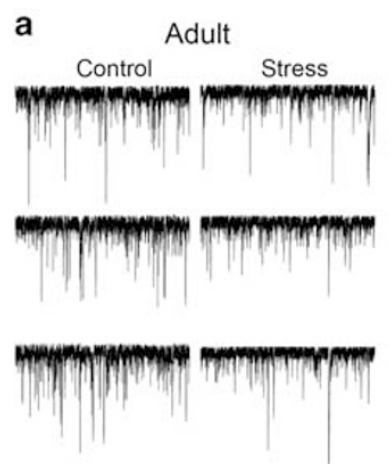

d

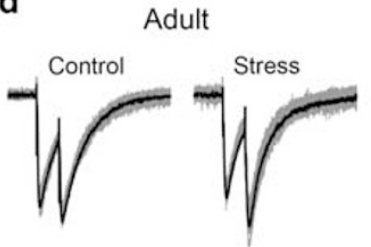

b

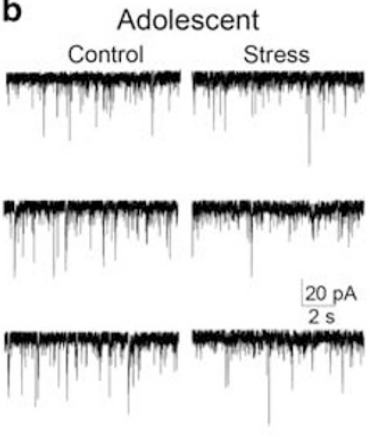

e

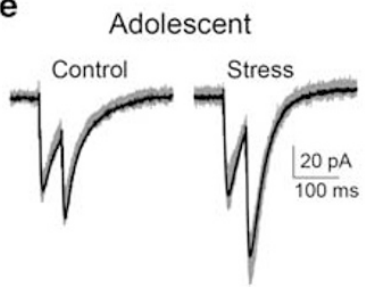

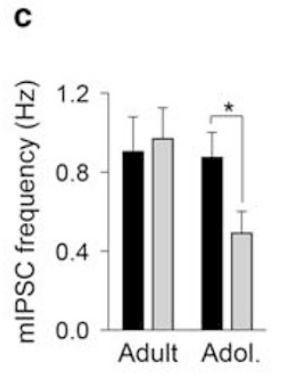

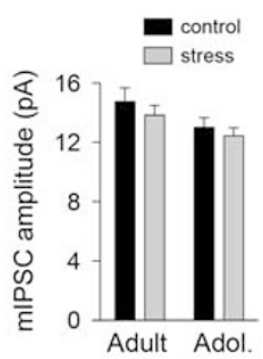

Figure 3 Repeated stress decreases $\boldsymbol{\gamma}$-aminobutyric acid (GABA) release in adolescents. Miniature inhibitory postsynaptic currents (mIPSCs) reflect release from GABAergic terminals, independent of GABA interneuron firing. mIPSCs were measured from lateral nucleus (LAT) principal neurons ex vivo with the addition of tetrodotoxin citrate (TTX). (a) Repeated stress had no apparent effect on the frequency of mIPSCs in these examples from adult rats. (b) Repeated stress decreased the frequency of mIPSCs in these examples from adolescent rats. (c) Repeated stress significantly decreased the frequency of mIPSCs in adolescent rats ( $* 0<0.05$, Holm-Sidak's multiple comparisons test after significance in two-way analysis of variance (ANOVA) but not in adult rats. Repeated stress had no significant effect on the amplitude of mIPSCs ( $p>0.05$ two-way ANOVA). (d) As further evidence for a presynaptic effect of repeated stress on GABA release, paired-pulse ratio was measured. Repeated stress had minimal impact on the relative amplitude of paired pulses in these examples from adult rats (gray: overlay of 10 traces; black: average of traces). (e) Repeated stress shifted the relative amplitude of paired pulses in these examples from adolescent rats. (f) Repeated stress significantly increased the paired-pulse ratio in adolescent rats $(* p<0.05$, Holm-Sidak's multiple comparisons test after significance in two-way ANOVA) but not in adult rats.

multiple comparisons test) but not in adult rats (Figures $3 \mathrm{a}$ and c; control $0.90 \pm 0.18 \mathrm{~Hz}, \quad n=27$ neurons; stress $0.97 \pm 0.16 \mathrm{~Hz}, n=24$ neurons; $p>0.05$, Holm-Sidak's multiple comparisons test). There was no significant effect of stress on the amplitude of mIPSCs (Figure 3c; main effect of stress $\mathrm{F}(1,109)=0.12, \quad p=0.73$, two-way ANOVA). A decrease of LAT GABAergic interneuron firing would be expected to produce a decrease in the frequency of sIPSCs with no effect on mIPSCs, as observed in adults, whereas an impairment of presynaptic GABA release probability is expected to cause an additional decrease in mIPSC frequency, as observed in adolescents. This was verified by measuring paired-pulse facilitation of IPSCs, which reflects presynaptic release function. Repeated stress shifted the paired-pulse ratio of IPSCs (main effect of stress, $\mathrm{F}(1,46)=7.278, p=0.0097)$, but this was significant only in adolescent rats (Figures $3 \mathrm{e}$ and f; $p<0.05$, Holm-Sidak's multiple comparisons test) and not in adult rats (Figures $3 \mathrm{~d}$ and f; $p>0.05$, Holm-Sidak's multiple comparisons test). These data indicate a dysfunction in GABAergic regulation of LAT after repeated stress.

\section{Different Effects of Stress on GABAergic Regulation of Adolescent Neurons In Vivo}

Differences observed in vitro set a background that can translate into differences of LAT neuronal firing in vivo. To test whether repeated stress changes the effects of GABA on LAT in vivo neuronal firing, local microiontophoresis was performed. LAT neurons were induced to fire at $5-6 \mathrm{~Hz}$ by continuous local iontophoretic glutamate application (no significant difference in glutamate-induced average firing rate across groups; adult control $5.9 \pm 0.5 \mathrm{~Hz}, n=28$ neurons, adult stress $6.2 \pm 0.3 \mathrm{~Hz}, n=47$ neurons, adolescent control $5.5 \pm 0.5 \mathrm{~Hz}, n=29$ neurons, adolescent stress $5.8 \pm 0.4 \mathrm{~Hz}$, $n=38$ neurons; main effect of stress $\mathrm{F}(1,103)=0.53, p=0.47$; main effect of age $\mathrm{F}(1,103)=0.78, \quad p=0.38$; stress $\times$ age interaction $\mathrm{F}(1,103)=0.0, p=0.95)$. Coiontophoresis of GABA suppressed glutamate-induced firing of LAT projection neurons in a dose-dependent manner in adolescent and adult rats (Figures $4 \mathrm{a}$ and $\mathrm{b}$; main effect of iontophoretic current intensity, adolescent $\mathrm{F}(4,260)=68.12, p<0.001$; adult $\mathrm{F}(4$, $292)=154.60, p<0.001$, two-way repeated-measure ANOVA). Repeated stress decreased the response of LAT neurons to GABA in adolescent rats (Figure $4 \mathrm{~b}$; main effect of stress, $\mathrm{F}(1,65)=8.29, p<0.01$, two-way repeated-measure ANOVA; $p<0.05$ at $20-30 \mathrm{nA}$, Holm-Sidak's multiple comparison test; there was a trend towards an apparent excitatory effect of GABA at low current intensities that could reflect indirect effects of GABA, for instance, effects on presynaptic release). In adult rats, repeated restraint did not significantly impact the sensitivity of LAT neuron firing to GABA (Figure 4a; no main effect of stress, $\mathrm{F}(1,64)=1.855, p=0.178$; stress $\times$ current intensity interaction, $F(4,256)=2.237$, $p=0.066$, two-way repeated-measures ANOVA). Because the data can be interpreted as a trend for an effect of repeated stress on sensitivity of LAT neuron firing to GABA in adults, the magnitude of the effects of stress were compared between adults and adolescents (see Materials and Methods section). There was a greater effect of repeated stress on GABA 

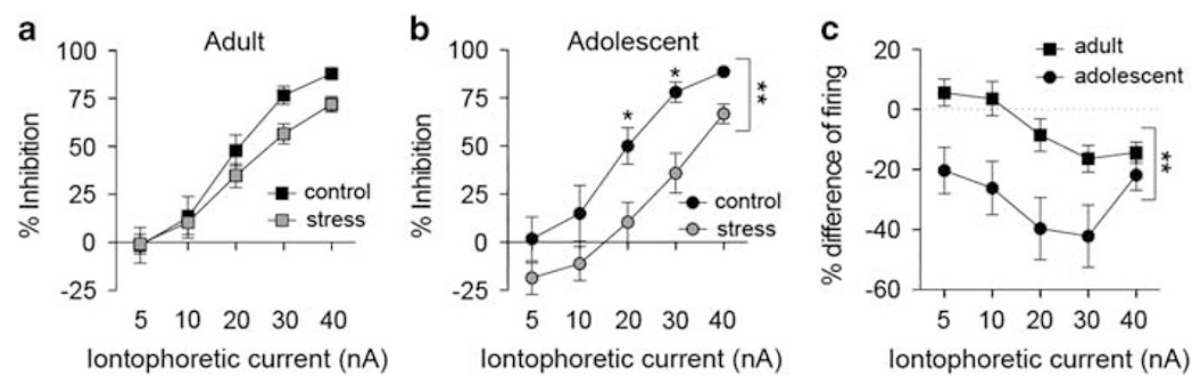

Figure 4 Repeated stress impairs the lateral nucleus (LAT) neuronal response to $\gamma$-aminobutyric acid (GABA) in adolescent rats. LAT neurons were induced to fire at $\sim 5 \mathrm{~Hz}$ with iontophoretic application of glutamate. Inhibition caused by coiontophoretic application of GABA was measured in vivo (5-40 nA dose-response). (a) In adult rats, repeated stress had no significant effect on the response of LAT neurons to iontophoretic GABA. (b) In adolescent rats, repeated stress significantly weakened the effect of iontophoretic GABA on LAT neuron firing $(* p<0.05$, Holm-Sidak's multiple comparisons test after significance in a two-way repeated-measures analysis of variance (ANOVA). (c) The magnitude of the effect of stress on GABA-induced inhibition was significantly greater in adolescent rats (*** $<0.05$ in a two-way repeated-measures ANOVA).
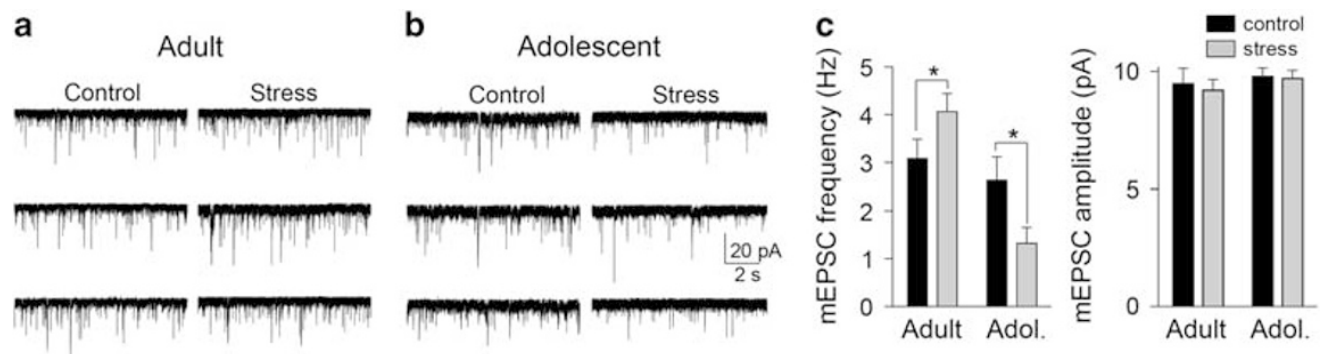

Figure 5 Repeated stress had different effects on miniature excitatory postsynaptic currents (EPSCs) in adult and adolescent rats. Miniature EPSCs (mEPSCs) were measured from lateral nucleus (LAT) principal neurons ex vivo to assess the effects of repeated stress on glutamatergic input. (a) Repeated stress increased the frequency of mEPSCs in these examples from adult rats. (b) Repeated stress decreased the frequency of mEPSCs in these examples from adolescent rats. (c) Repeated stress significantly increased the frequency of mEPSCs in adult rats but decreased the frequency of mEPSCs in adolescent rats (left; * $p<0.05$, Holm-Sidak's multiple comparisons test after significance in two-way analysis of variance (ANOVA), with no significant effect on mEPSC amplitude (right; no significance in two-way ANOVA).

sensitivity in adolescent rats than in adult rats (Figure 4c; main effect of age, $\mathrm{F}(1,83)=9.2, p=0.003$; age $\times$ iontophoretic current intensity interaction $\mathrm{F}(4,332)=2.7, p=0.03$; significant differences $5-30 \mathrm{nA}, p<0.05$, Holm-Sidak's multiple comparisons test). This indicates that repeated stress reduces the effects of GABA on LAT neuron firing in adolescent rats.

\section{Glutamatergic Drive does not Underlie Effects of Repeated Stress on LAT Neurons in Adolescents}

Glutamatergic input is a significant driver of LAT neuron firing. Enhanced response to glutamate may contribute to the effects of stress on LAT neuron firing. Therefore, the effects of repeated stress on glutamatergic transmission in vitro and glutamatergic driving of LAT neuron firing in vivo was measured. To test whether repeated stress exerts agedependent effects on glutamatergic synaptic inputs, mEPSCs were measured with in vitro whole-cell recordings. There was a significant effect of stress that depended on the age of the rat (stress $\times$ age interaction $\mathrm{F}(1,72)=5.9, p=0.02$, twoway ANOVA), with an increased frequency of mEPSCs in adult rats after repeated stress (Figures $5 \mathrm{a}$ and $\mathrm{c}$; control $3.1 \pm 0.3 \mathrm{~Hz}, n=17$ neurons; stress $4.2 \pm 0.4 \mathrm{~Hz}, \quad n=19$ neurons; $p<0.05$, Holm-Sidak's multiple comparisons test), but a significant decrease in the frequency of mEPSCs in adolescent rats after repeated stress (Figures $5 b$ and c; control
$2.6 \pm 0.5 \mathrm{~Hz}, \quad n=20$ neurons; stress $1.3 \pm 0.3 \mathrm{~Hz}, \quad n=20$ neurons; $p<0.05$, Holm-Sidak's multiple comparisons test). There was no significant effect of stress on the amplitude of mEPSCs (Figure $5 c$, right; main effect of stress $\mathrm{F}(1,72)=0.15$, $p=0.70$ ). These results demonstrate that repeated stress increases excitatory drive to LAT neurons in adult but not in adolescent rats.

We next measured the in vivo response of LAT neurons to iontophoretic glutamate. Repeated stress decreased the amount of glutamate needed to induce $5 \mathrm{~Hz}$ firing (Figure 6a; measured as iontophoretic current to induce $5 \mathrm{~Hz}$ firing; main effect of stress $\mathrm{F}(1,103)=15.3, p=0.0002$; stress $\times$ age interaction $\mathrm{F}(1,103)=4.4, p=0.04)$, but only in adult rats (adult control significantly greater than adult stress, $p<0.05$; adolescent control not significantly greater than adolescent stress, $p>0.05$, Holm-Sidak's multiple comparisons test).

To more thoroughly assess this effect of stress, a doseresponse of glutamate-induced firing was measured (10-40 nA, $20 \mathrm{~s}$ pulses). While adult and adolescent controls responded similarly to iontophoretic glutamate (main effect of age $\mathrm{F}(1,73)=0.02, p=0.88$; age $\times$ iontophoretic current intensity interaction $\mathrm{F}(3,219)=0.84, p=0.48$, two-way repeated-measures ANOVA), repeated stress increased glutamate-induced firing (main effect of stress $F(1,73)=8.3, p=0.005$; stress $\times$ iontophoretic current intensity interaction $\mathrm{F}(3,219)=2.6$, 
a

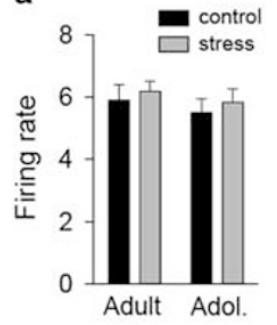

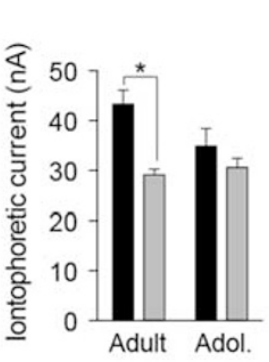

b

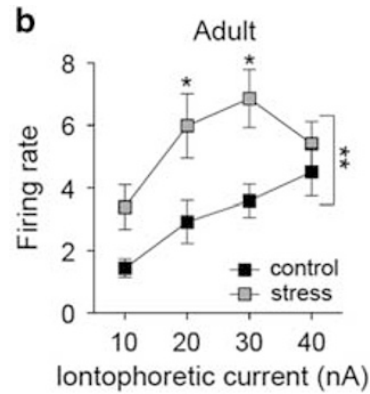

C

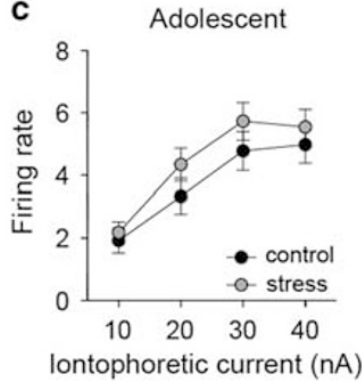

Figure 6 Repeated stress enhances the lateral nucleus (LAT) neuronal response to glutamate in adult rats. (a) Approximately 5-6 Hz firing rate of LAT neurons was induced by iontophoresis of glutamate (left). Repeated stress decreased the amount of iontophoretic glutamate needed to induce $5 \mathrm{~Hz}$ firing in adult rats but not in adolescent rats (right; $* 2<0.05$, Holm-Sidak's multiple comparisons test after significance in a two-way analysis of variance (ANOVA). (b) In adult rats, repeated stress significantly enhanced the LAT neuronal firing response to iontophoretic glutamate ( $10-40$ nA dose-response; * $p<0.05$, HolmSidak's multiple comparisons test after significance in a two-way repeated measures ANOVA). (c) In adolescent rats, there was no significant effect of repeated stress on glutamate-induced firing ( $10-40 \mathrm{nA}$ dose-response; no significance in two-way repeated-measures ANOVA).

$p=0.05$, two-way repeated-measures ANOVA), but only in adult rats (Figure 6b; 20-30 nA, $p<0.05$, Holm-Sidak's multiple comparisons test; control $n=20$, stress $n=18$ ), and not in adolescent rats (Figure $6 c ; p>0.05$ at all doses, HolmSidak's multiple comparisons test; control $n=18$, stress $n=19)$. This is consistent with increased excitatory effects of glutamate after repeated stress in adult but not in adolescent rats. In addition, because there was no evidence for increased excitatory drive in the adolescent LAT, these results indicate that increased excitatory drive is unlikely to underlie the effects of stress on LAT neuronal firing in adolescent rats.

\section{Stress Decreases the Firing of LAT Interneurons}

These data are suggestive of a reduction of GABAergic regulation in LAT after repeated stress. GABAergic interneurons are a primary source of GABA in the LAT. Therefore, the firing of LAT presumptive GABAergic interneurons was measured. Action potential half-width has been successfully used to separate interneurons and projection neurons. When the distribution of action potential half-widths was measured, evidence for a population of neurons with short-duration action potentials was uncovered (Figures 7a and b; data best fit with two curves compared with one curve; $p=0.012, F(6,86)=2.950$, extra sum-ofsquares F-test). Presumptive interneurons (from the narrowwidth action potential population, $<0.205 \mathrm{~ms}$ half-width) were analyzed separately. Repeated stress decreased the firing rate of these presumptive interneurons in adolescents (Figure 7c; main effect of stress, $\mathrm{F}(1,34)=8.151, p=0.0073$, two-way ANOVA; control $5.9 \pm 1.0 \mathrm{~Hz}, n=10$ neurons; stress $2.7 \pm 0.7 \mathrm{~Hz}, n=9$ neurons; $p<0.05$, Holm-Sidak's multiple comparisons test) but not reaching significance in adults (control $7.0 \pm 1.1 \mathrm{~Hz}, \quad n=10$ neurons; stress $4.8 \pm 0.8 \mathrm{~Hz}, n=9$ neurons; $p>0.05$, Holm-Sidak's multiple comparisons test). To further decrease the possibility that different populations of neurons were sampled between control and stress groups, the firing rate was plotted by halfwidth for each putative interneuron (Figure 7d). If a different population of neurons were sampled in control (those with a higher firing rate) and stress groups (those with a lower firing rate), a correlation would be expected between firing rate and half-width. There was no significant correlation (linear fit, $R^{2}=0.038, \mathrm{~F}(1,36)=1.409, p=0.243$ ).

\section{Stress Increases LAT Activity in Adolescents by Decreased GABAergic Inhibition}

The data presented above demonstrate that repeated stress has a greater effect on GABAergic inhibition in adolescents in vitro, and link the effects of stress on GABA with the effects of stress on LAT neuron firing in vivo. To test directly whether the effects of repeated stress on LAT neuron firing is due to altered GABAergic function, in vivo LAT neuron firing was recorded while a $\mathrm{GABA}_{\mathrm{A}}$ antagonist was infused into the LAT.

Intra-LAT infusion of the $\mathrm{GABA}_{\mathrm{A}}$ antagonist PTX mimicked the effects of repeated stress on LAT neuron activity in adolescents (Figures $8 \mathrm{~b}$ and d). PTX increased the number of active LAT neurons in adolescent rats (Figure 8d; age and PTX factor, main effect of PTX $\mathrm{F}(1,66)=5.9$, $p=0.018$, two-way ANOVA, age and PTX factors; significant age $\times$ PTX interaction $\mathrm{F}(1,66)=4.1, p=0.05$; adolescent baseline compared with PTX, $p<0.05$, Holm-Sidak's multiple comparisons test, $n=20$ vehicle, $n=13$ PTX) but not in adult rats (Figure $8 \mathrm{c}$; adult baseline compared with PTX, $p<0.05$, Holm-Sidak's multiple comparisons test, $n=20$ vehicle, $n=17$ PTX).

However, PTX also mimicked the effects of repeated stress on LAT neuron firing in adult rats, increasing the firing rate of neurons in the LAT in adult rats (Figure 8a; main effect of PTX $\mathrm{F}(1,15)=21.6, p=0.0003$, two-way repeated-measures ANOVA, age and PTX treatment factors; significant age $\times$ PTX interaction $\mathrm{F}(1,15)=13.3, p=0.002$; adult baseline compared with PTX, $p<0.05$, Holm-Sidak's multiple comparisons test, $n=10$ ) but not in adolescent rats (Figure 8b; adolescent baseline compared with PTX, $p>0.05$, Holm-Sidak's multiple comparisons test, $n=8)$. ACSF vehicle had no significant effect on firing rate (main effect of ACSF $\mathrm{F}(1,15)=1.2, p=0.29$, two-way repeatedmeasures ANOVA) or number of neurons/track (main effect of $\operatorname{ACSF} F(1,38)=0.15, p=0.7$, two-way repeated-measures ANOVA).

The effects of PTX in control rats indicate that decreased GABA could mimic the effects of repeated stress in both 
a

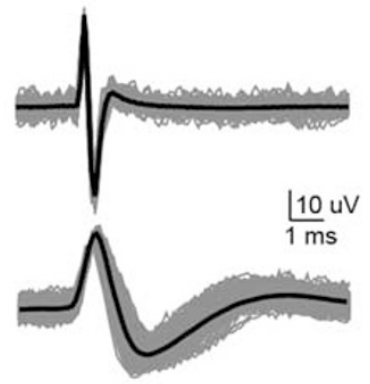

b

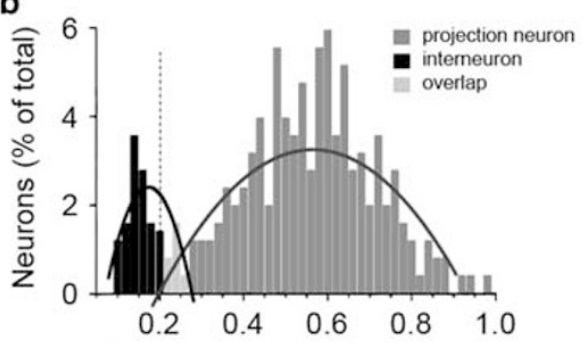

Action potential half-width (ms)

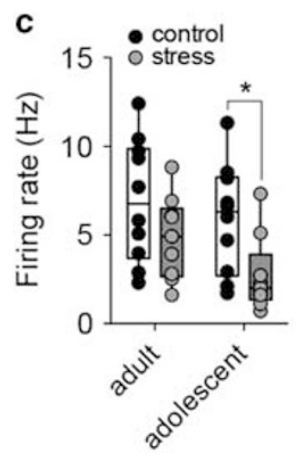

d

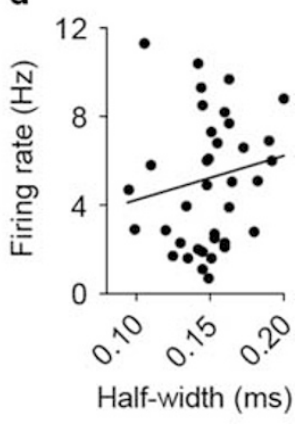

a
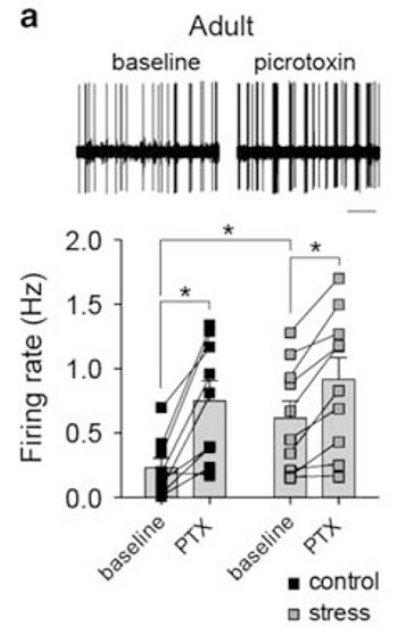

C

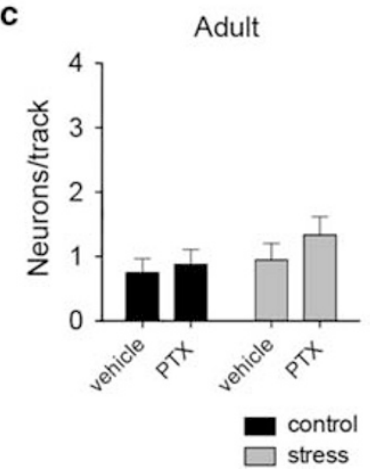

b
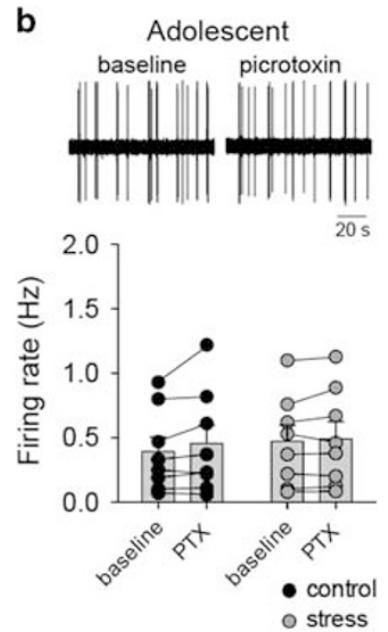

d

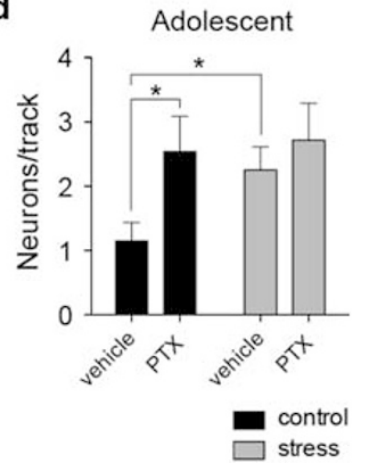

Figure 8 Repeated stress impairs in vivo GABAergic regulation of lateral nucleus (LAT) neurons in adolescent rats. To test whether differences of GABA underlie effects of stress on in vivo LAT neuron firing activity, picrotoxin (PTX) was infused into the LAT during electrophysiological recordings. (a) In adult rats, PTX infusion caused an increase of LAT neuron firing (top, representative extracellular recording trace of the same neuron before and after PTX). This was observed in control (left) and stress (right) conditions $(* p<0.05$, Holm-Sidak's multiple comparisons test after significance in a two-way repeated-measures analysis of variance (ANOVA). (b) In adolescent rats, PTX infusion did not significantly increase the firing rate of LAT neurons (top, representative extracellular recording trace of the same neuron before and after PTX; no significance in two-way repeatedmeasures ANOVA). (c) In adult rats, the number of active LAT neurons encountered per electrode track was not modified by PTX in control (left) or stress (right) conditions (no significance in two-way ANOVA). (d) In adolescent rats, PTX increased the number of active LAT neurons encountered in control conditions (left; $* p<0.05$, Holm-Sidak's multiple comparisons test after significance in a two-way ANOVA) but not in stress conditions (right, $p>0.05$, Holm-Sidak's multiple comparisons test after significance in a two-way ANOVA).

vehicle, $n=17$ PTX) or in the repeated stress group $(p<0.05$ Holm-Sidak's multiple comparison test; $n=20$ vehicle, $n=12$ PTX), such that stress and control groups were still significantly different after PTX. Because stress and control groups still differed when $\mathrm{GABA}_{\mathrm{A}}$ receptors were blocked, these results indicate that $\mathrm{GABA}_{\mathrm{A}}$ receptors do not underlie the measured effects of stress on LAT neuron activity in adult rats.

In contrast, PTX was significantly less effective in adolescent rats after repeated stress (Figure 8d; active neurons/electrode track, stress and PTX treatment factors, main effect of PTX $\mathrm{F}(1,63)=4.6, p=0.035$, two-way ANOVA), consistent with reduced GABAergic regulation 
of LAT neurons after repeated stress. Specifically, PTX did not increase the number of neurons active in adolescent rats exposed to stress ( $p>0.05$, Holm-Sidak's multiple comparisons test PTX in stress group; $n=20$ vehicle, $n=14$ PTX) but did increase this parameter in adolescent controls $(p<0.05$ Holm-Sidak's multiple comparisons test PTX in the control group; $n=20$ vehicle, $n=14$ PTX), such that stress and control adolescent groups were similar after PTX. This indicates that GABA has a necessary role in the expression of the effects of stress on LAT neuron activity in adolescent rats.

\section{DISCUSSION}

Repeated stress increases firing of LAT neurons in adult and adolescent rats. However, manifestation of this LAT hyperactivation after repeated stress is age-dependent, and may be due to distinct mechanisms at different ages. LAT projection neuron excitability is tightly controlled by GABAergic inhibition. Repeated stress modified GABAergic inhibition of LAT neurons prominently in adolescents but minimally in adults. Repeated stress reduced sensitivity to GABA in vivo, reduced GABAergic regulation of firing in vivo, and reduced synaptic sources of GABAergic inhibition in vitro in adolescent rats (Table 1). In addition, control and stress groups were equalized by a GABAergic antagonist, but only in adolescents. In contrast, repeated stress increased the sensitivity to glutamate in vivo and increased glutamatergic drive in vitro in adult rats (Table 1). The current experiments were performed in male rats. Therefore, one significant limitation of these findings is that they might not apply to females.

The GABAergic system in the BLA is still developing during the first several weeks postnatal. In rodents, the GABAergic interneuron network and basic GABAergic IPSC kinetics do not reach maturity until PND 14-28 (Berdel and Morys, 2000; Legaz et al, 2005; Olmos et al, 2005; Davila et al, 2008; Ehrlich et al, 2013). GABA also appears to exert differences in inhibitory regulation at later ages. Iontophoretic GABA suppressed glutamate-evoked firing of LAT projection neurons in adult and adolescent rats. However, in adolescent rats, blockade of the GABAergic system by picrotoxin resulted in an increase in the total number of active LAT neurons encountered, while in adult rats it resulted in an increase in firing rate. This demonstrates that ambient GABA may exert different influences on firing activity of LAT neurons in adolescent and adult rats. One interpretation of these data is that LAT neurons in adult rats are under somewhat uniform GABAergic regulation. When GABA receptors are blocked, these neurons will increase their firing rate. In contrast, LAT neurons in adolescent rats are under variable GABAergic regulation, such that some are under substantial GABAergic regulation, while others are under little GABAergic regulation. Those under substantial GABAergic inhibition are minimally active, whereas the remaining neurons are under little GABAergic inhibition and are active. The active neurons that are under little GABAergic inhibition are more likely to be sampled during baseline extracellular recordings. Because these active neurons are under little GABAergic inhibition, blockade of GABA receptors in adolescent rats will have minimal
Table I Summary of the Effects of Stress

\begin{tabular}{lcc}
\hline Measure & \multicolumn{2}{c}{ Effects of stress } \\
\cline { 2 - 3 } & Adult & Adolescent \\
\hline In vivo firing rate & $\uparrow$ & - \\
In vivo neurons/track & - & $\uparrow$ \\
sIPSC (Hz) & $\downarrow$ & $\downarrow$ \\
mIPSC (Hz) and release & - & $\downarrow$ \\
GABA sensitivity & - & $\downarrow$ \\
Interneuron firing rate & - & $\downarrow$ \\
mEPSC frequency & $\uparrow$ & $\downarrow$ \\
Glutamate sensitivity & $\uparrow$ & - \\
\hline
\end{tabular}

Effects of PTX

\begin{tabular}{lcccc}
\cline { 2 - 5 } & Control & Stress & Control & Stress \\
\hline In vivo firing rate & $\uparrow$ & $\uparrow$ & - & - \\
In vivo neurons/track & - & - & $\uparrow$ & - \\
\hline
\end{tabular}

Abbreviations: EPSC, excitatory postsynaptic current; GABA, $\gamma$-aminobutyric acid; mIPSC, miniature inhibitory postsynaptic current; sIPSC, spontaneous inhibitory postsynaptic current.

influence on their firing (Figure 8). Neurons that are under heavy GABAergic influence will display increased firing that will be observed as an increase in the number of active neurons in adolescent rats (Figure 8). Consistent with this, the number of active LAT neurons is higher in adolescent rats compared with adults, and there is substantially higher variability in the baseline firing rate of LAT neurons in adolescent rats (Figure 1). It is unclear why blockade of GABA receptors does not cause an increase in the number of active LAT neurons in adult rats (although there is a trend in this direction). Possible explanations may include additional sources of regulation (eg greater contribution of $h$ channels, SK channels, $\mathrm{GABA}_{\mathrm{b}}$ receptors; Ehrlich et al, 2012; Bosch and Ehrlich, 2015) that suppress neuron firing even when $\mathrm{GABA}_{\mathrm{A}}$ receptors are blocked.

Repeated stress increases the number of neurons active in adolescent rats but increases the firing rate of neurons in adult rats (Zhang and Rosenkranz, 2012). However, repeated stress only impaired GABAergic regulation in adolescents but not in adults. This dissociation implies that impaired GABAergic regulation of the LAT contributes to the effects of stress in adolescent rats, while other factors contribute more heavily in adult rats. In adolescents, our results point to impaired function of interneurons coupled with postsynaptic reduced sensitivity to GABA after repeated stress. Impaired function of LAT interneurons is reflected by decreased frequency of sIPSCs and mIPSCs and decreased activity of LAT presumptive GABAergic interneurons. In adults, only decreased sIPSCs was observed, consistent with a decrease in the ex vivo firing of LAT GABAergic interneurons. Previous studies hint towards impaired function of BLA interneurons after stress. A single stress experience reduces feedback inhibition (Isoardi et al, 2007) and depresses the interaction between GABAergic and glutamatergic input (Rodriguez Manzanares et al, 2005) in the adult BLA. Repeated stress 
also leads to decreased responsiveness of BLA interneurons, as measured by c-Fos and GABA efflux in response to acute stress (Reznikov et al, 2008; Reznikov et al, 2009), reduces GAD67, and induces dendritic atrophy of BLA interneurons (Gilabert-Juan et al, 2011). Repeated stress applied to juvenile rats can lead to changes of GABA function in adult rats, such as reduced GAD (Tzanoulinou et al, 2014) as well as changes observed as juveniles, such as reduced 5-HT and noradrenergic facilitation of sIPSCs (Braga et al, 2004; Jiang et al, 2009). However, the current study is the first to demonstrate directly the age bias in the effects of stress on GABAergic regulation of LAT neurons. In rats, BLA parvalbumin-positive interneurons reach maturity at $\sim$ PND 30 (Berdel and Morys, 2000; Legaz et al, 2005; Davila et al, 2008), which overlapped with the start of our repeated restraint protocol during adolescence. Repeated stress may stunt the final phases of prepubertal GABAergic development leading to greater impact of stress on GABAergic systems in adolescence. Despite the ex vivo change in LAT interneurons function indicated by decreased frequency of sIPSCs in adults, in vivo manipulation of GABAergic inhibition by PTX demonstrated that this change, or a change in GABAergic inhibition overall, is unlikely to explain the effects of stress on firing rate in adult rats.

Repeated stress reduced sensitivity to iontophoretic GABA in adolescents. This may be due to alterations of $\mathrm{GABA}_{\mathrm{A}}$ receptor subunit composition. Indeed, stress exposure can induce significant alteration in $\mathrm{GABA}_{\mathrm{A}}$ receptor subunit expression in the amygdala (Caldji et al, 2003; Jacobson-Pick and Richter-Levin, 2012; Jacobson-Pick et al, 2012; Tzanoulinou et al, 2014). Reduced sensitivity to GABA combined with impaired function of LAT interneurons may contribute to the observed reduction of ongoing GABAergic inhibition of the LAT. Reduced sensitivity to GABA might be expected to also lead to decreased mIPSC amplitude. Although trending in this direction, this was not a significant effect. Though speculative, this may imply that the reduced sensitivity to GABA is more prominent at extrasynaptic sites captured by iontophoretic application of GABA and local infusion of GABA antagonists. This is consistent with a previous finding of reduced tonic current in adolescent LAT neurons after repeated stress (Liu et al, 2014).

Other stress-induced changes could contribute to the observed increase of LAT projection neuron firing in adult rats. For instance, increased excitatory responses to glutamate and increased glutamatergic synaptic transmission. The increased excitatory response to glutamate could be caused by increased neuronal membrane excitability (Rosenkranz et al, 2010; Hetzel and Rosenkranz, 2014), increased function of NMDA or AMPA receptors (Adamec et al, 2005; Caudal et al, 2010; Mozhui et al, 2010; Suvrathan et al, 2014), reduced glutamatergic drive of GABAergic networks (Masneuf et al, 2014), or upregulation of glutamatergic receptors (Lei and Tejani-Butt, 2010; Gan et al, 2014). Increased glutamatergic synaptic transmission could be caused by increased glutamatergic inputs, as observed here and in other studies (Mitra et al, 2005; Vyas et al, 2006; Padival et al, 2013, 2015; Suvrathan et al, 2014; Tsai et al, 2014). In contrast, there was little evidence for increased glutamatergic drive after repeated stress in adolescent rats. In fact, a decrease of mEPSC frequency was observed, consistent with recent findings that repeated stress decreases the number of spines on LAT neurons in adolescent rats (Tsai et al, 2014; Padival et al, 2015).

Overall, repeated stress led to changes in the adolescent LAT that implicate impaired pre- and postsynaptic function of the GABAergic system. Reduced firing of GABAergic interneurons paired with decreased mIPSC frequency and increased PPR all indicate reduced release of GABA in the LAT. In combination with this, reduced sensitivity to iontophoretic GABA and reduced impact of picrotoxin is consistent with impaired postsynaptic inhibition by GABA. However, this impaired postsynaptic GABA may be specific for sensitivity to extrasynaptic GABA, as the amplitude of mIPSCs, which reflects synaptic GABA receptors, was not impacted by repeated stress.

The firing of projection neurons is also sensitive to neuromodulators. Amygdala GABAergic interneurons are targets of dopamine, NE, serotonin, acetylcholine, and cannabinoids (Brinley-Reed and McDonald, 1999; Rainnie, 1999; Katona et al, 2001; McDonald and Mascagni, 2001; Azad et al, 2003; Bissiere et al, 2003; Kroner et al, 2005; Muller et al, 2007, 2011; Tully et al, 2007; Pinard et al, 2008). Repeated stress may influence amygdala GABAergic transmission by impairing the neuromodulation of amygdala neurons (Braga et al, 2004; Buffalari and Grace, 2009; Hill et al, 2009, 2010), perhaps in an age-dependent manner.

Following repeated stress, abnormally high neuronal activity in conjunction with impaired GABAergic regulation is associated with a hyperactive and hyper-responsive amygdala. Studies have repeatedly demonstrated that the increased activity of the amygdala in adult male rats after stress parallels increased anxiety (Vyas et al, 2004; DoremusFitzwater et al, 2009; Rosenkranz et al, 2010) and production of behaviors that rely on the BLA, such as such as freezing in response to a fear-conditioned cue (Conrad et al, 1999; Rau et al, 2005; Rodriguez Manzanares et al, 2005; ToledoRodriguez and Sandi, 2007; Atchley et al, 2012; Zhang and Rosenkranz, 2013). Relatively less is known about the effects of stress on amygdala-dependent behaviors in adolescents; however, in several studies increased fear conditioning is accompanied by an impairment of fear extinction in adolescents and enhanced generalization of fear (Morrissey et al, 2011; Zhang and Rosenkranz, 2013; Muller et al, 2014; Padival et al, 2015). Both of these processes are sensitive to GABA within the BLA (Davis and Myers, 2002; Shaban et al, 2006; Lin et al, 2009; Sangha et al, 2009; Makkar et al, 2010; Lange et al, 2014). Similarly, impaired GABAergic systems may contribute to inappropriate affective responses characteristic of depression and anxiety.

Although there are few studies that examine the effects of repeated stress on amygdala function during adolescence, several studies test the impact of postweaning social isolation on BLA function. Consistent with the current results, postweaning social isolation, which shares features of chronic stress during adolescence, causes an increase of behaviors, or reduced extinction of behaviors, which rely on the BLA, such as learned fear responses to cues (Lukkes et al, 2009; Skelly et al, 2015; Walasek et al, 2002), but see (Morrissey et al, 2011). It also increases the responsiveness of neurons across the BLA in c-Fos studies (Toth et al, 2012), increases NMDA receptors (Gan et al, 2014), and increases the excitability of neurons in the basal nucleus (Rau et al, 2015), although the LAT has not yet been studied. However, 
particularly relevant to the current findings, postweaning social isolation has been found to decrease some measures of inhibition in the LAT (Lukkes et al, 2012).

Although adults and adolescents display amygdala hyperactivity after chronic stress, different mechanisms underlie this hyperactivity. Overall, these data indicate divergent mechanisms for stress-induced hyperactivation of the LAT across age; impaired GABAergic regulation in adolescent rats and increased glutamatergic excitation in adult rats. These findings may contribute to the age-dependent impact of stress on amygdala-mediated behaviors and affective disorders, and contribute to the development of age-appropriate treatment for these disorders.

\section{FUNDING AND DISCLOSURE}

Dr Zhang and Dr Rosenkranz report no biomedical financial interests or potential conflicts of interest.

\section{ACKNOWLEDGMENTS}

We gratefully acknowledge the help of Mallika Padival in histological processing of tissue. Grant support was provided by the National Institutes of Health (MH084970 and MH100536). The sponsors had no role in study design, collection, analysis and interpretation of data, writing of the report, or in the decision to submit this article for publication. A portion of the data was previously presented at the 2013 Annual Society for Neuroscience conference.

\section{REFERENCES}

Adamec R, Blundell J, Burton P (2005). Role of NMDA receptors in the lateralized potentiation of amygdala afferent and efferent neural transmission produced by predator stress. Physiol Behav 86: 75-91.

Allen JL, Lavallee KL, Herren C, Ruhe K, Schneider S (2010). DSMIV criteria for childhood separation anxiety disorder: informant, age, and sex differences. J Anxiety Disord 24: 946-952.

Almeida-Suhett CP, Prager EM, Pidoplichko V, Figueiredo TH, Marini AM, Li Z et al (2014). Reduced GABAergic inhibition in the basolateral amygdala and the development of anxiety-like behaviors after mild traumatic brain injury. PLoS One 9: e102627.

Atchley D, Hankosky ER, Gasparotto K, Rosenkranz JA (2012). Pharmacological enhancement of calcium-activated potassium channel function reduces the effects of repeated stress on fear memory. Behav Brain Res 232: 37-43.

Azad SC, Eder M, Marsicano G, Lutz B, Zieglgansberger W, Rammes G (2003). Activation of the cannabinoid receptor type 1 decreases glutamatergic and GABAergic synaptic transmission in the lateral amygdala of the mouse. Learn Mem 10: 116-128.

Beardslee WR, Gladstone TR, O'Connor EE (2012). Developmental risk of depression: experience matters. Child Adolesc Psychiatr Clin N Am 21: 261-278, vii.

Berdel B, Morys J (2000). Expression of calbindin-D28k and parvalbumin during development of rat's basolateral amygdaloid complex. Int J Dev Neurosci 18: 501-513.

Bissiere S, Humeau Y, Luthi A (2003). Dopamine gates LTP induction in lateral amygdala by suppressing feedforward inhibition. Nat Neurosci 6: 587-592.

Bosch D, Ehrlich I (2015). Postnatal maturation of GABAergic modulation of sensory inputs onto lateral amygdala principal neurons. J Physiol 593: 4387-4409.
Braga MF, Aroniadou-Anderjaska V, Manion ST, Hough CJ, Li H (2004). Stress impairs alpha(1A) adrenoceptor-mediated noradrenergic facilitation of GABAergic transmission in the basolateral amygdala. Neuropsychopharmacology 29: 45-58.

Bremne JD, Vermetten E (2001). Stress and development: behavioral and biological consequences. Dev Psychopathol 13: 473-489.

Bremner JD (2003). Long-term effects of childhood abuse on brain and neurobiology. Child Adolesc Psychiatr Clin N Am 12: 271-292.

Brinley-Reed M, McDonald AJ (1999). Evidence that dopaminergic axons provide a dense innervation of specific neuronal subpopulations in the rat basolateral amygdala. Brain Res 850: 127-135.

Buffalari DM, Grace AA (2009). Chronic cold stress increases excitatory effects of norepinephrine on spontaneous and evoked activity of basolateral amygdala neurons. Int J Neuropsychopharmacol 12: 95-107.

Caldji C, Diorio J, Meaney MJ (2003). Variations in maternal care alter $\mathrm{GABA}(\mathrm{A})$ receptor subunit expression in brain regions associated with fear. Neuropsychopharmacology 28: 1950-1959.

Carlson GA, Kashani JH (1988). Phenomenology of major depression from childhood through adulthood: analysis of three studies. Am J Psychiatry 145: 1222-1225.

Caudal D, Godsil BP, Mailliet F, Bergerot D, Jay TM (2010). Acute stress induces contrasting changes in AMPA receptor subunit phosphorylation within the prefrontal cortex, amygdala and hippocampus. PLoS One 5: e15282.

Chareyron LJ, Lavenex PB, Lavenex P (2012). Postnatal development of the amygdala: a stereological study in rats. J Comp Neurol 520: 3745-3763.

Conrad CD, LeDoux JE, Magarinos AM, McEwen BS (1999). Repeated restraint stress facilitates fear conditioning independently of causing hippocampal CA3 dendritic atrophy. Behav Neurosci 113: 902-913.

Copeland WE, Wolke D, Angold A, Costello EJ (2013). Adult psychiatric outcomes of bullying and being bullied by peers in childhood and adolescence. JAMA Psychiatry 70: 419-426.

Dahl RE (2001). Affect regulation, brain development, and behavioral/emotional health in adolescence. CNS Spectr 6: 60-72.

Davila JC, Olmos L, Legaz I, Medina L, Guirado S, Real MA (2008). Dynamic patterns of colocalization of calbindin, parvalbumin and GABA in subpopulations of mouse basolateral amygdalar cells during development. J Chem Neuroanat 35: 67-76.

Davis M, Myers KM (2002). The role of glutamate and gammaaminobutyric acid in fear extinction: clinical implications for exposure therapy. Biol Psychiatry 52: 998-1007.

Doremus TL, Brunell SC, Varlinskaya EI, Spear LP (2003). Anxiogenic effects during withdrawal from acute ethanol in adolescent and adult rats. Pharmacol Biochem Behav 75: 411-418.

Doremus TL, Varlinskaya EI, Spear LP (2004). Age-related differences in elevated plus maze behavior between adolescent and adult rats. Ann N Y Acad Sci 1021: 427-430.

Doremus-Fitzwater TL, Varlinskaya EI, Spear LP (2009). Social and non-social anxiety in adolescent and adult rats after repeated restraint. Physiol Behav 97: 484-494.

Ehrlich DE, Ryan SJ, Hazra R, Guo JD, Rainnie DG (2013). Postnatal maturation of GABAergic transmission in the rat basolateral amygdala. J Neurophysiol 110: 926-941.

Ehrlich DE, Ryan SJ, Rainnie DG (2012). Postnatal development of electrophysiological properties of principal neurons in the rat basolateral amygdala. J Physiol 590: 4819-4838.

Gan JO, Bowline E, Lourenco FS, Pickel VM (2014). Adolescent social isolation enhances the plasmalemmal density of NMDA NR1 subunits in dendritic spines of principal neurons in the basolateral amygdala of adult mice. Neuroscience 258: 174-183.

Gilabert-Juan J, Castillo-Gomez E, Perez-Rando M, Molto MD, Nacher J (2011). Chronic stress induces changes in the structure 
of interneurons and in the expression of molecules related to neuronal structural plasticity and inhibitory neurotransmission in the amygdala of adult mice. Exp Neurol 232: 33-40.

Grillon C, Duncko R, Covington MF, Kopperman L, Kling MA (2007). Acute stress potentiates anxiety in humans. Biol Psychiatry 62: 1183-1186.

Hetzel A, Rosenkranz JA (2014). Distinct effects of repeated restraint stress on basolateral amygdala neuronal membrane properties in resilient adolescent and adult rats. Neuropsychopharmacology 39: 2114-2130.

Hill MN, McLaughlin RJ, Bingham B, Shrestha L, Lee TT, Gray JM et al (2010). Endogenous cannabinoid signaling is essential for stress adaptation. Proc Natl Acad Sci USA 107: 9406-9411.

Hill MN, McLaughlin RJ, Morrish AC, Viau V, Floresco SB, Hillard CJ et al (2009). Suppression of amygdalar endocannabinoid signaling by stress contributes to activation of the hypothalamic-pituitary-adrenal axis. Neuropsychopharmacology 34: 2733-2745.

Isoardi NA, Bertotto ME, Martijena ID, Molina VA, Carrer HF (2007). Lack of feedback inhibition on rat basolateral amygdala following stress or withdrawal from sedative-hypnotic drugs. Eur J Neurosci 26: 1036-1044.

Jacobson-Pick S, Audet MC, McQuaid RJ, Kalvapalle R, Anisman H (2012). Stressor exposure of male and female juvenile mice influences later responses to stressors: modulation of GABAA receptor subunit mRNA expression. Neuroscience 215: 114-126.

Jacobson-Pick S, Richter-Levin G (2012). Short- and long-term effects of juvenile stressor exposure on the expression of GABAA receptor subunits in rats. Stress 15: 416-424.

Jiang X, Xing G, Yang C, Verma A, Zhang L, Li H (2009). Stress impairs 5-HT2A receptor-mediated serotonergic facilitation of GABA release in juvenile rat basolateral amygdala. Neuropsychopharmacology 34: 410-423.

Katona I, Rancz EA, Acsady L, Ledent C, Mackie K, Hajos N et al (2001). Distribution of CB1 cannabinoid receptors in the amygdala and their role in the control of GABAergic transmission. J Neurosci 21: 9506-9518.

Kessler RC, Birnbaum H, Bromet E, Hwang I, Sampson N, Shahly V (2010). Age differences in major depression: results from the National Comorbidity Survey Replication (NCS-R). Psychol Med 40: 225-237.

Kroner S, Rosenkranz JA, Grace AA, Barrionuevo G (2005). Dopamine modulates excitability of basolateral amygdala neurons in vitro. J Neurophysiol 93: 1598-1610.

Lang EJ, Pare D (1997). Similar inhibitory processes dominate the responses of cat lateral amygdaloid projection neurons to their various afferents. J Neurophysiol 77: 341-352.

Lange MD, Jungling K, Paulukat L, Vieler M, Gaburro S, Sosulina L et al (2014). Glutamic acid decarboxylase 65: a link between GABAergic synaptic plasticity in the lateral amygdala and conditioned fear generalization. Neuropsychopharmacology 39: 2211-2220.

Legaz I, Olmos L, Real MA, Guirado S, Davila JC, Medina L (2005). Development of neurons and fibers containing calcium binding proteins in the pallial amygdala of mouse, with special emphasis on those of the basolateral amygdalar complex. J Comp Neurol 488: 492-513.

Lei Y, Tejani-Butt SM (2010). N-methyl-D-aspartic acid receptors are altered by stress and alcohol in Wistar-Kyoto rat brain. Neuroscience 169: 125-131.

Likhtik E, Pelletier JG, Popescu AT, Pare D (2006). Identification of basolateral amygdala projection cells and interneurons using extracellular recordings. J Neurophysiol 96: 3257-3265.

Lin HC, Mao SC, Gean PW (2009). Block of gamma-aminobutyric acid-A receptor insertion in the amygdala impairs extinction of conditioned fear. Biol Psychiatry 66: 665-673.

Liu ZP, Song C, Wang M, He Y, Xu XB, Pan HQ et al (2014). Chronic stress impairs GABAergic control of amygdala through suppressing the tonic GABAA receptor currents. Mol Brain 7: 32.
Lukkes JL, Burke AR, Zelin NS, Hale MW, Lowry CA (2012). Postweaning social isolation attenuates c-Fos expression in GABAergic interneurons in the basolateral amygdala of adult female rats. Physiol Behav 107: 719-725.

Lukkes JL, Mokin MV, Scholl JL, Forster GL (2009). Adult rats exposed to early-life social isolation exhibit increased anxiety and conditioned fear behavior, and altered hormonal stress responses. Horm Behav 55: 248-256.

Lupien SJ, McEwen BS, Gunnar MR, Heim C (2009). Effects of stress throughout the lifespan on the brain, behaviour and cognition. Nat Rev Neurosci 10: 434-445.

Makkar SR, Zhang SQ, Cranney J (2010). Behavioral and neural analysis of GABA in the acquisition, consolidation, reconsolidation, and extinction of fear memory. Neuropsychopharmacology 35: $1625-1652$.

Masneuf S, Lowery-Gionta E, Colacicco G, Pleil KE, Li C, Crowley $\mathrm{N}$ et al (2014). Glutamatergic mechanisms associated with stress-induced amygdala excitability and anxiety-related behavior. Neuropharmacology 85: 190-197.

McDonald AJ (1982). Neurons of the lateral and basolateral amygdaloid nuclei: a Golgi study in the rat. J Comp Neurol 212: 293-312.

McDonald AJ (1992). Projection neurons of the basolateral amygdala: a correlative Golgi and retrograde tract tracing study. Brain Res Bull 28: 179-185.

McDonald AJ, Mascagni F (2001). Localization of the CB1 type cannabinoid receptor in the rat basolateral amygdala: high concentrations in a subpopulation of cholecystokinin-containing interneurons. Neuroscience 107: 641-652.

Mitra R, Jadhav S, McEwen BS, Vyas A, Chattarji S (2005). Stress duration modulates the spatiotemporal patterns of spine formation in the basolateral amygdala. Proc Natl Acad Sci USA 102: 9371-9376.

Morrissey MD, Mathews IZ, McCormick CM (2011). Enduring deficits in contextual and auditory fear conditioning after adolescent, not adult, social instability stress in male rats. Neurobiol Learn Mem 95: 46-56.

Mozhui K, Karlsson RM, Kash TL, Ihne J, Norcross M, Patel S et al (2010). Strain differences in stress responsivity are associated with divergent amygdala gene expression and glutamate-mediated neuronal excitability. J Neurosci 30: 5357-5367.

Muller I, Obata K, Richter-Levin G, Stork O (2014). GAD65 haplodeficiency conveys resilience in animal models of stressinduced psychopathology. Front Behav Neurosci 8: 265.

Muller JF, Mascagni F, McDonald AJ (2007). Serotoninimmunoreactive axon terminals innervate pyramidal cells and interneurons in the rat basolateral amygdala. J Comp Neurol 505: 314-335.

Muller JF, Mascagni F, McDonald AJ (2011). Cholinergic innervation of pyramidal cells and parvalbumin-immunoreactive interneurons in the rat basolateral amygdala. J Comp Neurol 519: 790-805.

National Research Council (US) Committee for the Update of the Guide for the Care and Use of Laboratory Animals. National Academies Press (US): Washington (DC), 2011.

Olmos JL, Real MA, Medina L, Guirado S, Davila JC (2005). Distribution of nitric oxide-producing neurons in the developing and adult mouse amygdalar basolateral complex. Brain Res Bull 66: 465-469.

Padival MA, Blume SR, Rosenkranz JA (2013). Repeated restraint stress exerts different impact on structure of neurons in the lateral and basal nuclei of the amygdala. Neuroscience 246: 230-242.

Padival MA, Blume SR, Vantrease JE, Rosenkranz JA (2015). Qualitatively different effect of repeated stress during adolescence on principal neuron morphology across lateral and basal nuclei of the rat amygdala. Neuroscience 291C: 128-145.

Paxinos G, Watson C (2008). The Rat Brain in Stereotaxic Coordinates: Compact, 6th edn. Academic Press: New York, NY. 
Pechtel P, Pizzagalli DA (2011). Effects of early life stress on cognitive and affective function: an integrated review of human literature. Psychopharmacology (Berl) 214: 55-70.

Pidoplichko VI, Aroniadou-Anderjaska V, Prager EM, Figueiredo TH, Almeida-Suhett CP, Miller SL (2014). ASICla activation enhances inhibition in the basolateral amygdala and reduces anxiety. J Neurosci 34: 3130-3141.

Pinard CR, Muller JF, Mascagni F, McDonald AJ (2008). Dopaminergic innervation of interneurons in the rat basolateral amygdala. Neuroscience 157: 850-863.

Rainnie DG (1999). Serotonergic modulation of neurotransmission in the rat basolateral amygdala. J Neurophysiol 82: 69-85.

Rainnie DG, Asprodini EK, Shinnick-Gallagher P (1991). Inhibitory transmission in the basolateral amygdala. J Neurophysiol 66: 999-1009.

Rainnie DG, Bergeron R, Sajdyk TJ, Patil M, Gehlert DR, Shekhar A (2004). Corticotrophin releasing factor-induced synaptic plasticity in the amygdala translates stress into emotional disorders. J Neurosci 24: 3471-3479.

Rao PA, Beidel DC, Turner SM, Ammerman RT, Crosby LE, Sallee FR (2007). Social anxiety disorder in childhood and adolescence: descriptive psychopathology. Behav Res Ther 45: 1181-1191.

Rau AR, Chappell AM, Butler TR, Ariwodola OJ, Weiner JL (2015). Increased basolateral amygdala pyramidal cell excitability may contribute to the anxiogenic phenotype induced by chronic earlylife stress. J Neurosci 35: 9730-9740.

Rau V, DeCola JP, Fanselow MS (2005). Stress-induced enhancement of fear learning: an animal model of posttraumatic stress disorder. Neurosci Biobehav Rev 29: 1207-1223.

Reznikov LR, Reagan LP, Fadel JR (2008). Activation of phenotypically distinct neuronal subpopulations in the anterior subdivision of the rat basolateral amygdala following acute and repeated stress. J Comp Neurol 508: 458-472.

Reznikov LR, Reagan LP, Fadel JR (2009). Effects of acute and repeated restraint stress on GABA efflux in the rat basolateral and central amygdala. Brain Res 1256: 61-68.

Rodriguez Manzanares PA, Isoardi NA, Carrer HF, Molina VA (2005). Previous stress facilitates fear memory, attenuates GABAergic inhibition, and increases synaptic plasticity in the rat basolateral amygdala. J Neurosci 25: 8725-8734.

Rosenkranz JA, Grace AA (1999). Modulation of basolateral amygdala neuronal firing and afferent drive by dopamine receptor activation in vivo. J Neurosci 19: 11027-11039.

Rosenkranz JA, Venheim ER, Padival M (2010). Chronic stress causes amygdala hyperexcitability in rodents. Biol Psychiatry 67: $1128-1136$.

Ryan SJ, Ehrlich DE, Jasnow AM, Daftary S, Madsen TE, Rainnie DG (2012). Spike-timing precision and neuronal synchrony are enhanced by an interaction between synaptic inhibition and membrane oscillations in the amygdala. PLoS One 7: e35320.

Sajdyk TJ, Shekhar A (1997). Excitatory amino acid receptor antagonists block the cardiovascular and anxiety responses elicited by gamma-aminobutyric acidA receptor blockade in the basolateral amygdala of rats. J Pharmacol Exp Ther 283: 969-977.

Sajdyk TJ, Shekhar A (2000). Sodium lactate elicits anxiety in rats after repeated GABA receptor blockade in the basolateral amygdala. Eur J Pharmacol 394: 265-273.

Sanders SK, Shekhar A (1995a). Anxiolytic effects of chlordiazepoxide blocked by injection of GABAA and benzodiazepine receptor antagonists in the region of the anterior basolateral amygdala of rats. Biol Psychiatry 37: 473-476.

Sanders SK, Shekhar A (1995b). Regulation of anxiety by GABAA receptors in the rat amygdala. Pharmacol Biochem Behav 52: 701-706.
Sangha S, Narayanan RT, Bergado-Acosta JR, Stork O, Seidenbecher T, Pape HC (2009). Deficiency of the $65 \mathrm{kDa}$ isoform of glutamic acid decarboxylase impairs extinction of cued but not contextual fear memory. J Neurosci 29: 15713-15720.

Shaban H, Humeau Y, Herry C, Cassasus G, Shigemoto R, Ciocchi S et al (2006). Generalization of amygdala LTP and conditioned fear in the absence of presynaptic inhibition. Nat Neurosci 9: $1028-1035$.

Skelly MJ, Chappell AE, Carter E, Weiner JL (2015). Adolescent social isolation increases anxiety-like behavior and ethanol intake and impairs fear extinction in adulthood: possible role of disrupted noradrenergic signaling. Neuropharmacology 97: 149-159.

Spear LP (2000). The adolescent brain and age-related behavioral manifestations. Neurosci Biobehav Rev 24: 417-463.

Suvrathan A, Bennur S, Ghosh S, Tomar A, Anilkumar S, Chattarji S (2014). Stress enhances fear by forming new synapses with greater capacity for long-term potentiation in the amygdala. Philos Trans R Soc Lond Ser B 369: 20130151.

Toledo-Rodriguez M, Sandi C (2007). Stress before puberty exerts a sex- and age-related impact on auditory and contextual fear conditioning in the rat. Neural Plast 2007: 71203.

Toth M, Tulogdi A, Biro L, Soros P, Mikics E, Haller J (2012). The neural background of hyper-emotional aggression induced by post-weaning social isolation. Behav Brain Res 233: 120-129.

Truitt WA, Johnson PL, Dietrich AD, Fitz SD, Shekhar A (2009). Anxiety-like behavior is modulated by a discrete subpopulation of interneurons in the basolateral amygdala. Neuroscience 160: 284-294.

Truitt WA, Sajdyk TJ, Dietrich AD, Oberlin B, McDougle CJ, Shekhar A (2007). From anxiety to autism: spectrum of abnormal social behaviors modeled by progressive disruption of inhibitory neuronal function in the basolateral amygdala in Wistar rats. Psychopharmacology (Berl) 191: 107-118.

Tsai SF, Huang TY, Chang CY, Hsu YC, Chen SJ, Yu L et al (2014). Social instability stress differentially affects amygdalar neuron adaptations and memory performance in adolescent and adult rats. Front Behav Neurosci 8: 27.

Tully K, Li Y, Tsvetkov E, Bolshakov VY (2007). Norepinephrine enables the induction of associative long-term potentiation at thalamo-amygdala synapses. Proc Natl Acad Sci USA 104: 14146-14150.

Tzanoulinou S, Garcia-Mompo C, Castillo-Gomez E, Veenit V, Nacher J, Sandi C (2014). Long-term behavioral programming induced by peripuberty stress in rats is accompanied by GABAergicrelated alterations in the Amygdala. PLoS One 9: e94666.

Vyas A, Jadhav S, Chattarji S (2006). Prolonged behavioral stress enhances synaptic connectivity in the basolateral amygdala. Neuroscience 143: 387-393.

Vyas A, Pillai AG, Chattarji S (2004). Recovery after chronic stress fails to reverse amygdaloid neuronal hypertrophy and enhanced anxiety-like behavior. Neuroscience 128: 667-673.

Walasek G, Wesierska M, Werka T (2002). Effects of social rearing conditions on conditioned suppression in rats. Acta Neurobiol Exp (Wars) 62: 25-31.

Zhang W, Hetzel A, Shah B, Atchley D, Blume SR, Padival MA et al (2014). Greater physiological and behavioral effects of interrupted stress pattern compared to daily restraint stress in rats. PLoS One 9: e102247.

Zhang W, Rosenkranz JA (2012). Repeated restraint stress increases basolateral amygdala neuronal activity in an agedependent manner. Neuroscience 226: 459-474.

Zhang W, Rosenkranz JA (2013). Repeated restraint stress enhances cue-elicited conditioned freezing and impairs acquisition of extinction in an age-dependent manner. Behav Brain Res 248: $12-24$. 